\title{
Fermionic Basis in Conformal Field Theory and Thermodynamic Bethe Ansatz for Excited States ${ }^{\star}$
}

\author{
Hermann BOOS $\ddagger$ \\ $\dagger$ Fachbereich C-Physik, Bergische Universität Wuppertal, 42097 Wuppertal, Germany \\ E-mail: boos@physik.uni-wuppertal.de \\ $\ddagger$ Skobeltsyn Institute of Nuclear Physics, Moscow State University, 119991 Moscow, Russia
}

Received October 07, 2010, in final form January 05, 2011; Published online January 13, 2011

doi:10.3842/SIGMA.2011.007

\begin{abstract}
We generalize the results of [Comm. Math. Phys. 299 (2010), 825-866] (hidden Grassmann structure IV) to the case of excited states of the transfer matrix of the sixvertex model acting in the so-called Matsubara direction. We establish an equivalence between a scaling limit of the partition function of the six-vertex model on a cylinder with quasi-local operators inserted and special boundary conditions, corresponding to particlehole excitations, on the one hand, and certain three-point correlation functions of conformal field theory (CFT) on the other hand. As in hidden Grassmann structure IV, the fermionic basis developed in previous papers and its conformal limit are used for a description of the quasi-local operators. In paper IV we claimed that in the conformal limit the fermionic creation operators generate a basis equivalent to the basis of the descendant states in the conformal field theory modulo integrals of motion suggested by A. Zamolodchikov (1987). Here we argue that, in order to completely determine the transformation between the above fermionic basis and the basis of descendants in the CFT, we need to involve excitations. On the side of the lattice model we use the excited-state TBA approach. We consider in detail the case of the descendant at level 8 .
\end{abstract}

Key words: integrable models; six vertex model; XXZ spin chain; fermionic basis, thermodynamic Bethe ansatz; excited states; conformal field theory; Virasoro algebra

2010 Mathematics Subject Classification: 82B20; 82B21; 82B23; 81T40; 81Q80

\section{Introduction}

Much progress was made in the understanding of the connection between the one-dimensional XXZ spin chain and two-dimensional quantum field theories (QFT). Many different aspects of this connection were studied in the literature. In the present paper we will touch only some particular aspect related to the hidden fermionic structure of the XXZ model and the corresponding continuum model - conformal field theory (CFT), [1, 2, 3, 4].

The continuum model can be studied through the scaling limit of the six-vertex model compactified on an infinite cylinder of radius $R$. The corresponding direction around the cylinder is sometimes called Matsubara direction. It is well known that the scaling limit of the homogeneous critical six-vertex model is related to CFT, while introducing inhomogeneities in a special way leads to the sine-Gordon model (sG) which has a mass gap, [5]. An important step forward was done by Bazhanov, Lukyanov and Zamolodchikov in papers [6, 7, 8]. They obtained an integrable structure of CFT by constructing a monodromy matrix with the quantum space related to the chiral bosonic field and the Heisenberg algebra. On the other hand this monodromy

\footnotetext{
${ }^{\star}$ This paper is a contribution to the Special Issue "Relationship of Orthogonal Polynomials and Special Functions with Quantum Groups and Integrable Systems". The full collection is available at http://www.emis.de/journals/SIGMA/OPSF.html
} 
matrix satisfies the Yang-Baxter equation with the $R$-matrix of the six-vertex model. The corresponding transfer matrix fulfills Baxter's $T Q$-relation [9] and generates the integrals of motion. These integrals of motion are special combinations of the Virasoro generators. Originally they were introduced by Alexander Zamolodchikov in [10]. Zamolodchikov observed that the integrals of motion generate that part of the Virasoro algebra that survives under the integrable $\Phi_{1,3}$-perturbation of the CFT.

In order to state the full equivalence of the six-vertex model in the scaling limit and the CFT one needs to compare all possible correlation functions. This problem is far from being solved completely. We believe that it can be helpful to use a hidden fermionic structure of the spin- $\frac{1}{2}$ XXZ chain, $[1,2]$. The key idea is to consider a fermionic basis generated by means of certain creation operators $\mathbf{t}^{*}, \mathbf{b}^{*}, \mathbf{c}^{*}$. These operators act on a space of quasi-local operators $\mathcal{W}^{(\alpha)}$. Any operator in this space can be represented in the form $q^{2 \alpha S(0)} \mathcal{O}$ with some operator $\mathcal{O}$ which acts on a chain segment of arbitrary finite length. The operator $q^{2 \alpha S(0)}$ was called "primary field" because it fulfills some properties similar to the properties of certain primary field in CFT. The fermionic basis is constructed inductively. Its completeness was shown in [11]. An important theorem proved by Jimbo, Miwa and Smirnov in the paper [3] allows one to reduce any correlation function expressed through the fermionic basis to determinants. An interesting feature of the above construction is that it is algebraic in the sense that the fermionic operators are constructed by means of the representation theory of the quantum group $U_{q}\left(\widehat{\mathfrak{s l}}_{2}\right)$. They are independent of any physical data like the magnetic field, the temperature, the boundary conditions etc. For example, the temperature can be incorporated via the Suzuki-Trotter formalism [12] by taking inhomogeneity parameters in the Matsubara direction in a special way and then performing the so-called Trotter limit, when the number of sites in the Matsubara direction $\mathbf{n} \rightarrow \infty$. One can even keep the number $\mathbf{n}$ finite and consider the case of arbitrary inhomogeneity parameters in this direction. The fermionic basis will not be affected [3]. There are only two transcendental functions $\rho$ and $\omega$ which "absorb" the whole physical information and appear in the determinants in analogy with the situation with free fermions. In [3] these functions were represented in terms of the differential of the second kind in the theory of deformed Abelian integrals for finite $\mathbf{n}$. These functions can be also obtained within the TBA approach, [13] which makes it possible to take the Trotter limit $\mathbf{n} \rightarrow \infty$. In the recent papers $[14,15]$ the fermionic basis was used in order to study the one-point functions of the sine-Gordon model on a cylinder. In this case the transcendental functions must be modified.

Coming back to the similarity with the CFT, let us emphasize that the construction of the fermionic basis bears some similarity with the construction of the descendants in CFT via the action of the Virasoro generators on the primary fields. In the paper [4] we tried to make this similarity more explicit. Namely, we related the main building blocks of CFT - the three-point correlators to the scaling limit of a special partition functions of the six-vertex model constructed with the help of the fermionic operators. More concretely, we believe that the following conjecture is true. There exist scaling limits $\boldsymbol{\tau}^{*}, \boldsymbol{\beta}^{*}, \boldsymbol{\gamma}^{*}$ of the operators $\mathbf{t}^{*}, \mathbf{b}^{*}, \mathbf{c}^{*}$ and scaling limits $\rho^{\text {sc }}$ and $\omega^{\text {sc }}$ of the functions $\rho$ and $\omega$ as well. The operator $\tau^{*}$ generates Zamolodchikov's integrals of motion mentioned above, while the asymptotic expansion of the function $\rho^{\mathrm{sc}}$ with respect to its spectral parameter generates their vacuum expectation values. Another conjecture was that the asymptotic expansion of $\omega^{\mathrm{sc}}$ with respect to the spectral parameters describes the expectation values of descendants for CFT with central charge $c=1-6 \nu^{2} /(1-\nu)$ where $\nu$ is related to the deformation parameter $q=e^{\pi i \nu}$. Equivalently, one takes the six-vertex model on a cylinder for some special boundary conditions and considers the scaling limit of the corresponding normalized partition function with inserted quasi-local operator $q^{2 \alpha S(0)} \mathcal{O}$. On the other hand one computes a normalized three-point function in the CFT on an infinite cylinder with Virasoro descendants of some primary field $\phi_{\alpha}$ of conformal dimension $\Delta_{\alpha}$ inserted at the 
origin and two primary fields $\phi_{ \pm}$inserted at $+\infty$ and $-\infty$ respectively. The conjecture states that for any quasi-local operator one can find a corresponding descendant in such a way that the above partition function and the CFT three-point function are equal. In order to state this equality we used the fermionic basis for the quasi-local operators mentioned above.

In [4] we established such a correspondence between the fermionic basis and the Virasoro generators only up to the level 6 . It corresponds to bilinear combinations of the "Fouriermodes" $\boldsymbol{\beta}_{j}^{*}$ and $\boldsymbol{\gamma}_{j}^{*}$ with odd index $j$. For level 8 we have 5 Virasoro descendants $\mathbf{l}_{-2}^{4}, \mathbf{l}_{-4} \mathbf{l}_{-2}^{2}$, $\mathbf{l}_{-4}^{2}, \mathbf{l}_{-6} \mathbf{l}_{-2}, \mathbf{l}_{-8}$. As for the fermionic basis, we also find five linearly independent combinations of the creation operators $\boldsymbol{\beta}_{j}^{*}$ and $\boldsymbol{\gamma}_{j}^{*}$. One of them is quartic: $\boldsymbol{\beta}_{1}^{*} \boldsymbol{\beta}_{3}^{*} \boldsymbol{\gamma}_{3}^{*} \boldsymbol{\gamma}_{1}^{*}$. Unfortunately, we could not uniquely fix the transformation matrix between the fermionic basis and the basis generated by the Virasoro descendants. We thought that it could be done if we would insert the simplest excitation corresponding to $L_{-1} \phi_{ \pm}$at $\pm \infty$ instead of the primary fields $\phi_{ \pm}$themselves. The difference between the "global" Virasoro generators $L_{n}$ and the "local" ones $\mathbf{l}_{n}$ was explained in [4]. It is also discussed in the next section.

The original motivation of the present paper was to fill this gap. It turned out, however, that it was not enough to consider only the simplest excitation. The above uncertainty still remained in this case. One has to take at least the next excitation, namely, that one corresponding to the descendants of the second level $L_{-1}^{2} \phi_{ \pm}$and $L_{-2} \phi_{ \pm}$in order to fix the unknown elements of the transformation matrix. All these elements are certain rational functions of the central charge $c$ and the conformal dimension of the primary field $\Delta_{\alpha} \cdot{ }^{1}$ They do not depend on the conformal dimensions $\Delta_{ \pm}$of the primary fields $\phi_{ \pm}$. They are also independent of the choice of the excitation at $\pm \infty$. This is rather strong condition. We are still unable to prove it for arbitrary excitation. We think that it is interesting to consider excitations also independently of the above concrete problem. Therefore we consider them in a more general setting and come to the solution of the level 8 problem in the very end.

The paper is organized as follows. In Section 2 we remind the reader about the main results of the paper [4]. Section 3 is devoted to the TBA approach for the excited states. We derive the equation for the auxiliary function $\Theta$ and define the function $\omega^{\text {sc }}$ in Section 4 . In Section 5 we discuss the relation to certain CFT three-point functions. We discuss the solution of the above level 8 problem in Section 6. In Appendix A we show several leading terms of the asymptotic expansion of the function $\Psi$ defined in Section 3 and discuss its relation to the integrals of motion. In Appendices $\mathrm{B}$ and $\mathrm{C}$ several leading terms for the asymptotic expansions of the functions $\bar{F}, x^{ \pm}, \Theta$ are explicitly shown.

\section{Reminder of basic results of [4]}

As was mentioned in the Introduction, in the paper [4] some specific connection between the conformal field theory (CFT) with the central charge $c=1-6 \nu^{2} /(1-\nu)$ and the XXZ model with the deformation parameter $q=e^{\pi i \nu}$ was established ${ }^{2}$. More precisely, the following relation was found to be valid with the left hand side containing the CFT data and the right hand side containing the lattice model data

$$
\frac{\left\langle\Delta_{-}\left|P_{\alpha}\left(\left\{\mathbf{l}_{-k}\right\}\right) \phi_{\alpha}(0)\right| \Delta_{+}\right\rangle}{\left\langle\Delta_{-}\left|\phi_{\alpha}(0)\right| \Delta_{+}\right\rangle}=\lim _{\mathbf{n} \rightarrow \infty, a \rightarrow 0, \mathbf{n} a=2 \pi R} Z^{\kappa, s}\left\{q^{2 \alpha S(0)} \mathcal{O}\right\} .
$$

The left hand side means a normalized three-point function of the CFT defined on a cylinder of radius $R$ parameterized by a complex variable $z=x+i y$ with spacial coordinate $x$ : $-\infty<x<\infty$

\footnotetext{
${ }^{1}$ Look at the formulae (12.4) of [4] or (2.18) and (6.9)-(6.11).

${ }^{2}$ Usually we take $\nu$ in the region $\frac{1}{2}<\nu<1$ called in [7] a "semi-classical domain". The region $0<\nu \leq \frac{1}{2}$ demands more accurate treatment.
} 
and coordinate in the Matsubara direction $y:-\pi R<y<\pi R$. The equivalence of the points $x \pm \pi i R$ is implied. At the origin $z=0$ some descendant field is inserted which is given by some polynomial $P_{\alpha}\left(\left\{\mathbf{1}_{-k}\right\}\right)$ of Virasoro generators $\mathbf{l}_{-k}, k>0$ acting on the primary field $\phi_{\alpha}(z)$ with conformal dimension

$$
\Delta_{\alpha}=\frac{\nu^{2} \alpha(\alpha-2)}{4(1-\nu)} .
$$

We called these Virasoro generators "local" in a sense that they are defined in vicinity of $z=0$ with the corresponding energy-momentum tensor

$$
T(z)=\sum_{n=-\infty}^{\infty} \mathbf{l}_{n} z^{-n-2}
$$

The bra- and ket-states $\left|\Delta_{+}\right\rangle$and $\left\langle\Delta_{-}\right|$are to be defined through two primary fields $\phi_{ \pm}$with conformal dimensions $\Delta_{ \pm}$being inserted at $x \rightarrow \pm \infty$ in such a way that $L_{n}\left|\Delta_{+}\right\rangle=\delta_{n, 0} \Delta_{+}\left|\Delta_{+}\right\rangle$, $n \geq 0$ when $x=\infty$ and $\left\langle\Delta_{-}\right| L_{n}=\delta_{n, 0} \Delta_{-}\left\langle\Delta_{-}\right|, n \leq 0$ at $x=-\infty$. We called the Virasoro generators $L_{n}$ "global". They correspond to the expansion obtained via the conformal transformation $z \rightarrow e^{-z / R}$

$$
T(z)=\frac{1}{R^{2}}\left(\sum_{n=-\infty}^{\infty} L_{n} e^{\frac{n z}{R}}-\frac{c}{24}\right) .
$$

In [10] Alexander Zamolodchikov introduced the local integrals of motion which act on local operators as

$$
\left(\mathbf{i}_{2 n-1} O\right)(w)=\int_{C_{w}} \frac{d z}{2 \pi i} h_{2 n}(z) O(w) \quad(n \geq 1),
$$

where the densities $h_{2 n}(z)$ are certain descendants of the identity operator $I$. An important property is that

$$
\left\langle\Delta_{-}\left|\mathbf{i}_{2 n-1}(O(z))\right| \Delta_{+}\right\rangle=\left(I_{2 n-1}^{+}-I_{2 n-1}^{-}\right)\left\langle\Delta_{-}|O(z)| \Delta_{+}\right\rangle,
$$

where $I_{2 n-1}^{ \pm}$denote the vacuum eigenvalues of the local integrals of motion on the Verma module with conformal dimension $\Delta_{ \pm}$. The Verma module is spanned by the elements

$$
\mathbf{i}_{2 k_{1}-1} \cdots \mathbf{i}_{2 k_{p}-1} \mathbf{l}_{-2 l_{1}} \cdots \mathbf{l}_{-2 l_{q}}\left(\phi_{\alpha}(0)\right) \text {. }
$$

In case when $\Delta_{+}=\Delta_{-}$the space is spanned by the even Virasoro generators $\left\{\mathbf{1}_{-2 n}\right\}_{n \geq 1}$.

In order to describe the right hand side of (2.1) we need the fermionic basis constructed in $[1,2]$ via certain creation operators. These creation operators called $\mathbf{t}^{*}, \mathbf{b}^{*}, \mathbf{c}^{*}$ together with the annihilation operators called $\mathbf{b}, \mathbf{c}$ act in the space ${ }^{3}$

$$
\mathcal{W}^{(\alpha)}=\bigoplus_{s=-\infty}^{\infty} \mathcal{W}_{\alpha-s, s}
$$

where $\mathcal{W}_{\alpha-s, s}$ is the subspace of quasi-local operators of the spin $s$ with the shifted $\alpha$-parameter. They all are defined as formal power series of $\zeta^{2}-1$ and have the block structure

$$
\mathbf{t}^{*}(\zeta): \quad \mathcal{W}_{\alpha-s, s} \rightarrow \mathcal{W}_{\alpha-s, s}
$$

\footnotetext{
${ }^{3}$ The problem of constructing the annihilation operator corresponding to the creation operator $\mathbf{t}^{*}$ was discussed in the paper [13] but was not solved completely.
} 


$$
\begin{aligned}
& \mathbf{b}^{*}(\zeta), \mathbf{c}(\zeta): \mathcal{W}_{\alpha-s+1, s-1} \rightarrow \mathcal{W}_{\alpha-s, s} \\
& \mathbf{c}^{*}(\zeta), \mathbf{b}(\zeta): \mathcal{W}_{\alpha-s-1, s+1} \rightarrow \mathcal{W}_{\alpha-s, s}
\end{aligned}
$$

The operator $\mathbf{t}^{*}(\zeta)$ plays the role of a generating function of the commuting integrals of motion. In a sense it is bosonic. It commutes with all fermionic operators $\mathbf{b}(\zeta), \mathbf{c}(\zeta)$ and $\mathbf{b}^{*}(\zeta), \mathbf{c}^{*}(\zeta)$ which obey canonical anti-commutation relations

$$
\left[\mathbf{c}(\xi), \mathbf{c}^{*}(\zeta)\right]_{+}=\psi(\xi / \zeta, \alpha), \quad\left[\mathbf{b}(\xi), \mathbf{b}^{*}(\zeta)\right]_{+}=-\psi(\zeta / \xi, \alpha)
$$

with

$$
\psi(\zeta, \alpha)=\frac{1}{2} \zeta^{\alpha} \frac{\zeta^{2}+1}{\zeta^{2}-1}
$$

The annihilation operators b and c "kill" the lattice "primary field" $q^{2 \alpha S(0)}$

$$
\mathbf{b}(\zeta)\left(q^{2 \alpha S(0)}\right)=0, \quad \mathbf{c}(\zeta)\left(q^{2 \alpha S(0)}\right)=0, \quad S(k)=\frac{1}{2} \sum_{j=-\infty}^{k} \sigma_{j}^{3} .
$$

The space of states is generated via the multiple action of the creation operators $\mathbf{t}^{*}(\zeta), \mathbf{b}^{*}(\zeta)$, $\mathbf{c}^{*}(\zeta)$ on the "primary field" $q^{2 \alpha S(0)}$. In this way one can obtain the fermionic basis. The completeness of this basis was proved in the paper [11].

In the right hand side of equation (2.1) we take the scaling limit of a normalized partition function of the six-vertex model on a cylinder with insertion of a quasi-local operator $q^{2 \alpha S(0)} \mathcal{O}$

$$
Z^{\kappa, s}\left\{q^{2 \alpha S(0)} \mathcal{O}\right\}=\frac{\operatorname{Tr}_{\mathbf{S}} \operatorname{Tr}_{\mathbf{M}}\left(Y_{\mathbf{M}}^{(-s)} T_{\mathrm{S}, \mathbf{M}} q^{2 \kappa S} \mathbf{b}_{\infty, s-1}^{*} \cdots \mathbf{b}_{\infty, 0}^{*}\left(q^{2 \alpha S(0)} \mathcal{O}\right)\right)}{\operatorname{Tr}_{\mathbf{S}} \operatorname{Tr}_{\mathbf{M}}\left(Y_{\mathbf{M}}^{(-s)} T_{\mathrm{S}, \mathbf{M}} q^{2 \kappa S} \mathbf{b}_{\infty, s-1}^{*} \cdots \mathbf{b}_{\infty, 0}^{*}\left(q^{2 \alpha S(0)}\right)\right)},
$$

where the operators $\mathbf{b}_{\infty, j}^{*}$ are defined through ${ }^{4}$ a singular part when $\zeta \rightarrow 0$ and $\mathbf{b}_{\text {reg }}^{*}$ is a regular one

$$
\zeta^{-\alpha} \mathbf{b}^{*}(\zeta)(X)=\sum_{j=0}^{s-1} \zeta^{-2 j} \mathbf{b}_{\infty, j}^{*}(X)+\zeta^{-\alpha} \mathbf{b}_{\mathrm{reg}}^{*}(\zeta)(X), \quad X \in \mathcal{W}_{\alpha-s+1, s-1}
$$

for some operator $X$ of the spin $s-1, s>0$ where $\zeta^{-\alpha} \mathbf{b}_{\text {reg }}^{*}(\zeta)(X)$ vanishes at zero. The monodromy matrix $T_{\mathrm{S}, \mathrm{M}}$ is defined via the universal $R$-matrix of $U_{q}\left(\widehat{\mathfrak{s l}}_{2}\right)$ on the tensor product of two evaluation representations where the first one $\mathfrak{H}_{\mathrm{S}}$ corresponds to the infinite lattice direction and the second one $\mathfrak{H}_{\mathbf{M}}$ corresponds to the Matsubara direction

$$
T_{\mathrm{S}, \mathbf{M}}=\prod_{j=-\infty}^{\curvearrowright} T_{j, \mathbf{M}}, \quad T_{j, \mathbf{M}} \equiv T_{j, \mathbf{M}}(1), \quad T_{j, \mathbf{M}}(\zeta)=\prod_{\mathbf{m}=\mathbf{1}}^{\curvearrowleft} L_{j, \mathbf{m}}(\zeta)
$$

with the standard $L$-operator of the six vertex model

$$
L_{j, \mathbf{m}}(\zeta)=q^{-\frac{1}{2} \sigma_{j}^{3} \sigma_{\mathbf{m}}^{3}}-\zeta^{2} q^{\frac{1}{2} \sigma_{j}^{3} \sigma_{\mathbf{m}}^{3}}-\zeta\left(q-q^{-1}\right)\left(\sigma_{j}^{+} \sigma_{\mathbf{m}}^{-}+\sigma_{j}^{-} \sigma_{\mathbf{m}}^{+}\right) .
$$

\footnotetext{
${ }^{4}$ Actually, later we discuss the Fateev-Dotsenko condition $(2.13)$ fulfilled for the case $\kappa^{\prime}=\kappa$. In this case the parameters $\alpha$ and $s$ are constrained. We will be mostly interested in the case when $0<\alpha<2$. Then we need $s<0$ and another definition of the functional (2.6) is necessary. It can be done through the replacement of operators $\mathbf{b}_{\infty, j}^{*}$ by $\mathbf{c}_{\infty, j}^{*}$. This choice was taken in [15] with identification of the notation there $\mathbf{c}_{\mathrm{screen},-j}^{*}=\mathbf{c}_{\infty, j}^{*}$.
} 
The "screening operator" $Y_{\mathbf{M}}^{(-s)}$ carries spin $-s$. As was discussed in [4], the functional (2.6) does not depend on the concrete choice of the screening operator under rather mild conditions. Due to common wisdom, in case of an infinite lattice, one can change boundary conditions and, instead of taking the traces in the right hand side of (2.6), insert two one-dimensional projectors $|\kappa\rangle\langle\kappa|$ and $|\kappa+\alpha-s, s\rangle\langle\kappa+\alpha-s, s|$ at the boundary, where $|\kappa\rangle$ is eigenvector of the transfer matrix $T_{\mathbf{M}}(\zeta, \kappa)=\operatorname{Tr}_{j}\left(T_{j, \mathbf{M}} q^{\kappa \sigma_{j}^{3}}\right)$ with maximal eigenvalue $T(\zeta, \kappa)$ in the zero spin sector and where the eigenvector $|\kappa+\alpha-s, s\rangle$ corresponds to the maximal eigenvalue $T(\zeta, \kappa+\alpha-s, s)$ of the transfer matrix $T_{\mathbf{M}}(\zeta, \kappa+\alpha-s)$ in the sector with spin $s$. The twist parameter $\kappa$ plays the role of the magnetic field. So, we can perform the following substitution

$$
Z^{\kappa, s}\left\{q^{2 \alpha S(0)} \mathcal{O}\right\} \rightarrow \frac{\left\langle\kappa+\alpha-s, s\left|T_{\mathrm{S}, \mathbf{M}} q^{2 \kappa S} \mathbf{b}_{\infty, s-1}^{*} \cdots \mathbf{b}_{\infty, 0}^{*}\left(q^{2 \alpha S(0)} \mathcal{O}\right)\right| \kappa\right\rangle}{\left\langle\kappa+\alpha-s, s\left|T_{\mathrm{S}, \mathbf{M}} q^{2 \kappa S} \mathbf{b}_{\infty, s-1}^{*} \cdots \mathbf{b}_{\infty, 0}^{*}\left(q^{2 \alpha S(0)}\right)\right| \kappa\right\rangle},
$$

which does not affect the answer for the case of infinite lattice if

$$
\left\langle\kappa\left|Y_{\mathbf{M}}^{(-s)}\right| \kappa+\alpha-s, s\right\rangle \neq 0 .
$$

The theorem proved by Jimbo, Miwa and Smirnov [3] claims ${ }^{5}$ that

$$
\begin{aligned}
& Z^{\kappa, s}\left\{\mathbf{t}^{*}(\zeta)(X)\right\}=2 \rho(\zeta \mid \kappa, \kappa+\alpha, s) Z^{\kappa, s}\{X\}, \\
& Z^{\kappa, s}\left\{\mathbf{b}^{*}(\zeta)(X)\right\}=\frac{1}{2 \pi i} \oint_{\Gamma} \omega(\zeta, \xi \mid \kappa, \alpha, s) Z^{\kappa, s}\{\mathbf{c}(\xi)(X)\} \frac{d \xi^{2}}{\xi^{2}}, \\
& Z^{\kappa, s}\left\{\mathbf{c}^{*}(\zeta)(X)\right\}=-\frac{1}{2 \pi i} \oint_{\Gamma} \omega(\xi, \zeta \mid \kappa, \alpha, s) Z^{\kappa, s}\{\mathbf{b}(\xi)(X)\} \frac{d \xi^{2}}{\xi^{2}},
\end{aligned}
$$

where the contour $\Gamma$ goes around all the singularities of the integrand except $\xi^{2}=\zeta^{2}$. The direct consequence of the above theorem and the anti-commutation relations (2.5) is the determinant formula

$$
\begin{gathered}
Z^{\kappa, s}\left\{\mathbf{t}^{*}\left(\zeta_{1}^{0}\right) \cdots \mathbf{t}^{*}\left(\zeta_{p}^{0}\right) \mathbf{b}^{*}\left(\zeta_{1}^{+}\right) \cdots \mathbf{b}^{*}\left(\zeta_{r}^{+}\right) \mathbf{c}^{*}\left(\zeta_{r}^{-}\right) \cdots \mathbf{c}^{*}\left(\zeta_{1}^{-}\right)\left(q^{2 \alpha S(0)}\right)\right\} \\
=\prod_{i=1}^{p} 2 \rho\left(\zeta_{i}^{0} \mid \kappa, \kappa+\alpha, s\right) \times \operatorname{det}\left(\omega\left(\zeta_{i}^{+}, \zeta_{j}^{-} \mid \kappa, \alpha, s\right)\right)_{i, j=1, \ldots, r} .
\end{gathered}
$$

The functions $\rho$ and $\omega$ are completely defined by the Matsubara data. The function $\rho$ is the ratio of two eigenvalues of the transfer matrix

$$
\rho(\zeta \mid \kappa+\alpha-s, s)=\frac{T(\zeta, \kappa+\alpha-s, s)}{T(\zeta, \kappa)} .
$$

We will come to the definition of the function $\omega$ in Section 4 in more general case of presence of the excited states.

The scaling limit in the Matsubara direction means

$$
\mathbf{n} \rightarrow \infty, \quad a \rightarrow 0, \quad \mathbf{n} a=2 \pi R,
$$

where the radius of the cylinder $R$ is fixed. Simultaneously one should rescale the spectral parameter

$$
\zeta=\lambda \bar{a}^{\nu}, \quad \bar{a}=C a
$$

\footnotetext{
${ }^{5}$ Actually, in [3] the statement was proved for the case $s=0$ but as was discussed in [4], this statement can be proved for $s \neq 0$ also.
} 
with some fine-tuning constant $C$. One of the most important points of [4] was to define the scaling limits of $\rho$ and $\omega$

$$
\begin{aligned}
& \rho^{\mathrm{sc}}\left(\lambda \mid \kappa, \kappa^{\prime}\right)=\lim _{\text {scaling }} \rho\left(\lambda \bar{a}^{\nu} \mid \kappa, \alpha, s\right), \\
& \omega^{\mathrm{sc}}\left(\lambda, \mu \mid \kappa, \kappa^{\prime}, \alpha\right)=\frac{1}{4} \lim _{\text {scaling }} \omega\left(\lambda \bar{a}^{\nu}, \mu \bar{a}^{\nu} \mid \kappa, \alpha, s\right)
\end{aligned}
$$

where $\kappa^{\prime}$ is defined through an analogue of the Dotsenko-Fateev condition [16]

$$
\kappa^{\prime}=\kappa+\alpha+2 \frac{1-\nu}{\nu} s .
$$

The continuum limit can be taken in both directions of the cylinder.

The first conjecture proposed in [4] was that the creation operators are well-defined in the scaling limit for the space direction when $j a=x$ is finite

$$
\boldsymbol{\tau}^{*}(\lambda)=\lim _{a \rightarrow 0} \frac{1}{2} \mathbf{t}^{*}\left(\lambda \bar{a}^{\nu}\right), \quad \boldsymbol{\beta}^{*}(\lambda)=\lim _{a \rightarrow 0} \frac{1}{2} \mathbf{b}^{*}\left(\lambda \bar{a}^{\nu}\right), \quad \boldsymbol{\gamma}^{*}(\lambda)=\lim _{a \rightarrow 0} \frac{1}{2} \mathbf{c}^{*}\left(\lambda \bar{a}^{\nu}\right)
$$

and for the "primary field"

$$
\Phi_{\alpha}(0)=\lim _{a \rightarrow 0} q^{2 \alpha S(0)} .
$$

Asymptotic expansions at $\lambda \rightarrow \infty$ look

$$
\begin{aligned}
& \log \left(\boldsymbol{\tau}^{*}(\lambda)\right) \simeq \sum_{j=1}^{\infty} \boldsymbol{\tau}_{2 j-1}^{*} \lambda^{-\frac{2 j-1}{\nu}} \\
& \frac{1}{\sqrt{\boldsymbol{\tau}^{*}(\lambda)}} \boldsymbol{\beta}^{*}(\lambda) \simeq \sum_{j=1}^{\infty} \boldsymbol{\beta}_{2 j-1}^{*} \lambda^{-\frac{2 j-1}{\nu}}, \quad \frac{1}{\sqrt{\boldsymbol{\tau}^{*}(\lambda)}} \boldsymbol{\gamma}^{*}(\lambda) \simeq \sum_{j=1}^{\infty} \boldsymbol{\gamma}_{2 j-1}^{*} \lambda^{-\frac{2 j-1}{\nu}} .
\end{aligned}
$$

The next conjecture based on the bosonisation arguments was that the scaling limit of the space $\mathcal{W}_{\alpha-s, s}$ belongs to the direct product of two Verma modules

$$
\text { Scaling limit }\left(\mathcal{W}_{\alpha-s, s}\right) \subset \mathcal{V}_{\alpha+2 \frac{1-\nu}{\nu} s} \otimes \overline{\mathcal{V}}_{-\alpha}
$$

and the operators $\boldsymbol{\tau}^{*}(\lambda), \boldsymbol{\beta}^{*}(\lambda), \boldsymbol{\gamma}^{*}(\lambda)$ act non-trivially only on the first chirality component and do not touch the second one

$$
\begin{aligned}
& \tau_{2 j-1}^{*}: V_{\alpha+2 \frac{1-\nu}{\nu} s} \rightarrow \mathcal{V}_{\alpha+2 \frac{1-\nu}{\nu} s}, \\
& \beta_{2 j-1}^{*}: V_{\alpha+2 \frac{1-\nu}{\nu}(s-1)} \rightarrow \mathcal{V}_{\alpha+2 \frac{1-\nu}{\nu} s}, \\
& \gamma_{2 j-1}^{*}: V_{\alpha+2 \frac{1-\nu}{\nu}(s+1)} \rightarrow V_{\alpha+2 \frac{1-\nu}{\nu} s} .
\end{aligned}
$$

Using the results of the paper [7], we get the asymptotic expansion for $\lambda \rightarrow+\infty$

$$
\log \rho^{\mathrm{sc}}\left(\lambda \mid \kappa, \kappa^{\prime}\right) \simeq \sum_{j=1}^{\infty} \lambda^{-\frac{2 j-1}{\nu}} C_{j}\left(I_{2 j-1}^{+}-I_{2 j-1}^{-}\right) .
$$

Here the integrals of motion $I_{2 j-1}^{ \pm}$are the same as in (2.3). They correspond to the "right" and the "left" vacuum and depend on $\kappa$ and $\kappa^{\prime}$ respectively:

$$
I_{2 j-1}^{+}=I_{2 j-1}(\kappa), \quad I_{2 j-1}^{-}=I_{2 j-1}\left(\kappa^{\prime}\right) .
$$


We can identify

$$
\boldsymbol{\tau}_{2 j-1}^{*}=C_{j} \mathbf{i}_{2 j-1} \text {. }
$$

For the function $\omega^{\mathrm{sc}}$ we have

$$
\omega^{\mathrm{sc}}\left(\lambda, \mu \mid \kappa, \kappa^{\prime}, \alpha\right) \simeq \sqrt{\rho^{\mathrm{sc}}\left(\lambda \mid \kappa, \kappa^{\prime}\right)} \sqrt{\rho^{\mathrm{sc}}\left(\mu \mid \kappa, \kappa^{\prime}\right)} \sum_{i, j=1}^{\infty} \lambda^{-\frac{2 i-1}{\nu}} \mu^{-\frac{2 j-1}{\nu}} \omega_{i, j}\left(\kappa, \kappa^{\prime}, \alpha\right)
$$

when $\lambda^{2}, \mu^{2} \rightarrow+\infty$.

The scaling limit of (2.8) is proportional to

$$
\begin{gathered}
Z_{R}^{\kappa, \kappa^{\prime}}\left\{\boldsymbol{\tau}^{*}\left(\lambda_{1}^{0}\right) \cdots \boldsymbol{\tau}^{*}\left(\lambda_{p}^{0}\right) \boldsymbol{\beta}^{*}\left(\lambda_{1}^{+}\right) \cdots \boldsymbol{\beta}^{*}\left(\lambda_{r}^{+}\right) \boldsymbol{\gamma}^{*}\left(\lambda_{r}^{-}\right) \cdots \boldsymbol{\gamma}^{*}\left(\lambda_{1}^{-}\right)\left(\Phi_{\alpha}(0)\right)\right\} \\
=\prod_{i=1}^{p} \rho^{\mathrm{sc}}\left(\lambda_{i}^{0} \mid \kappa, \kappa^{\prime}\right) \times \operatorname{det}\left(\omega^{\mathrm{sc}}\left(\lambda_{i}^{+}, \lambda_{j}^{-} \mid \kappa, \kappa^{\prime}, \alpha\right)\right)_{i, j=1, \ldots, r} .
\end{gathered}
$$

If we substitute the expansion (2.14) into the left hand side of (2.17) and the expansions (2.15), (2.16) into the right hand side of (2.17) we can compare the coefficients standing with powers of the spectral parameters and express the functional $Z_{R}^{\kappa, \kappa^{\prime}}$ of any monomial constructed from the modes $\boldsymbol{\tau}_{2 j-1}^{*}, \boldsymbol{\beta}_{2 j-1}^{*}, \boldsymbol{\gamma}_{2 j-1}^{*}$ through the integrals of motion $I_{2 n-1}$, coefficients $C_{n}$ and $\omega_{i, j}$. In [4] we argued that the eigenvalue $T(\zeta, \kappa+\alpha-s, s)$ in the scaling limit (2.10) becomes equal to $T\left(\zeta, \kappa^{\prime}\right)$. This means that, if we choose $\alpha$ and the spin $s$ in such a way that $\kappa^{\prime}=\kappa$, then using (2.9) and (2.12), we get $\rho^{\mathrm{sc}}\left(\lambda \mid \kappa, \kappa^{\prime}\right)=1$. This is an important technical point. In this case we were able to apply the Wiener-Hopf technique and obtain the coefficients $\omega_{i, j}$ as power series in $\kappa^{-1}$ where $\kappa \rightarrow \infty$.

On the other hand, one can evaluate the left hand side of (2.1) using the operator product expansion (OPE). In order to compare with the result obtained by the above lattice method, one needs to identify the parameters $\kappa, \kappa^{\prime}, \alpha$ with the CFT data. In fact, we already identified $\alpha$ by taking the formula (2.2) for the dimension $\Delta_{\alpha}$ of the primary field $\phi_{\alpha}$. The next step was to take $\Delta_{+}=\Delta_{\kappa+1}$ and $\Delta_{-}=\Delta_{-\kappa^{\prime}+1}$.

The most important conjecture of [4] was that for the CFT with central charge $c=1-6 \frac{\nu^{2}}{1-\nu}$ it is always possible to find one-to-one correspondence between a polynomial $P_{\alpha}\left(\left\{\mathbf{l}_{-k}\right\}\right)$ in the left hand side of (2.1) and certain combinations of $\boldsymbol{\beta}_{2 j-1}^{*}$ and $\boldsymbol{\gamma}_{2 j-1}^{*}$.

It is convenient to introduce

$$
\boldsymbol{\beta}_{2 m-1}^{*}=D_{2 m-1}(\alpha) \boldsymbol{\beta}_{2 m-1}^{\mathrm{CFT} *}, \quad \boldsymbol{\gamma}_{2 m-1}^{*}=D_{2 m-1}(2-\alpha) \boldsymbol{\gamma}_{2 m-1}^{\mathrm{CFT} *},
$$

where

$$
D_{2 m-1}(\alpha)=\frac{1}{\sqrt{i \nu}} \Gamma(\nu)^{-\frac{2 m-1}{\nu}}(1-\nu)^{\frac{2 m-1}{2}} \frac{1}{(m-1) !} \frac{\Gamma\left(\frac{\alpha}{2}+\frac{1}{2 \nu}(2 m-1)\right)}{\Gamma\left(\frac{\alpha}{2}+\frac{(1-\nu)}{2 \nu}(2 m-1)\right)}
$$

together with even and odd bilinear combinations

$$
\begin{aligned}
& \boldsymbol{\phi}_{2 m-1,2 n-1}^{\text {even }}=(m+n-1) \frac{1}{2}\left(\boldsymbol{\beta}_{2 m-1}^{\mathrm{CFT} *} \boldsymbol{\gamma}_{2 n-1}^{\mathrm{CFT} *}+\boldsymbol{\beta}_{2 n-1}^{\mathrm{CFT} *} \boldsymbol{\gamma}_{2 m-1}^{\mathrm{CFT} *}\right), \\
& \boldsymbol{\phi}_{2 m-1,2 n-1}^{\mathrm{odd}}=d_{\alpha}^{-1}(m+n-1) \frac{1}{2}\left(\boldsymbol{\beta}_{2 n-1}^{\mathrm{CFT} *} \boldsymbol{\gamma}_{2 m-1}^{\mathrm{CFT} *}-\boldsymbol{\beta}_{2 m-1}^{\mathrm{CFT} *} \boldsymbol{\gamma}_{2 n-1}^{\mathrm{CFT} *}\right),
\end{aligned}
$$

where

$$
d_{\alpha}=\frac{\nu(\nu-2)}{\nu-1}(\alpha-1)=\frac{1}{6} \sqrt{(25-c)\left(24 \Delta_{\alpha}+1-c\right)} .
$$


The Verma module has a basis consisting of the vectors (2.4). Conjecturally the same space is spanned by:

$$
\mathbf{i}_{2 k_{1}-1} \cdots \mathbf{i}_{2 k_{p}-1} \phi_{2 m_{1}-1,2 n_{1}-1}^{\text {even }} \cdots \phi_{2 m_{r}-1,2 n_{r}-1}^{\text {even }} \phi_{2 \bar{m}_{1}-1,2 \bar{n}_{1}-1}^{\text {odd }} \phi_{2 \bar{m}_{\bar{r}}-1,2 \bar{n}_{\bar{r}}-1}^{\text {odd }}\left(\phi_{\alpha}\right) .
$$

Since

$$
\left[\mathbf{l}_{0}, \boldsymbol{\tau}_{2 j-1}^{*}\right]=(2 j-1) \boldsymbol{\tau}_{2 j-1}^{*}, \quad\left[\mathbf{l}_{0}, \boldsymbol{\beta}_{2 i-1}^{*} \boldsymbol{\gamma}_{2 j-1}^{*}\right]=(2 i+2 j-2) \boldsymbol{\beta}_{2 i-1}^{*} \boldsymbol{\gamma}_{2 j-1}^{*},
$$

the descendants of the form (2.4) and those created by the fermions of the form (2.20) must be finite linear combinations of each other if the corresponding levels coincide

$$
\sum_{j=1}^{q} 2 l_{j}=\sum_{k=1}^{r} 2\left(m_{k}+n_{k}-1\right)+\sum_{\bar{k}=1}^{\bar{r}} 2\left(\bar{m}_{\bar{k}}+\bar{n}_{\bar{k}}-1\right) .
$$

As was discussed above, one can choose the parameters $\alpha$ and $s$ in such a way that $\kappa^{\prime}=\kappa$ and $\Delta_{+}=\Delta_{-}$. With this choice we factor out the integrals of motion. The quotient space of the Verma module modulo the action of the integrals of motion is spanned by the vectors of the form

$$
\mathbf{l}_{-2 l_{1}} \cdots \mathbf{l}_{-2 l_{q}}\left(\phi_{\alpha}(0)\right)
$$

All coefficients of the polynomial $P_{\alpha}\left(\left\{\mathbf{l}_{-k}\right\}\right)$ are independent of $\kappa$. We were able to identify the above basis vectors up to the level 6 . The system of equations is overdetermined but nevertheless it has a solution:

$$
\begin{aligned}
\phi_{1,1}^{\text {even }} \cong & \mathbf{l}_{-2}, \quad \phi_{1,3}^{\text {even }} \cong \mathbf{l}_{-2}^{2}+\frac{2 c-32}{9} \mathbf{l}_{-4}, \quad \phi_{1,3}^{\text {odd }} \cong \frac{2}{3} \mathbf{l}_{-4}, \\
\phi_{1,5}^{\text {even }} \cong & \mathbf{l}_{-2}^{3}+\frac{c+2-20 \Delta+2 c \Delta}{3(\Delta+2)} \mathbf{l}_{-4} \mathbf{l}_{-2} \\
& +\frac{-5600+428 c-6 c^{2}+2352 \Delta-300 c \Delta+12 c^{2} \Delta+896 \Delta^{2}-32 c \Delta^{2}}{60(\Delta+2)} \mathbf{l}_{-6}, \\
\phi_{1,5}^{\text {odd }} \cong & \frac{2 \Delta}{\Delta+2} \mathbf{l}_{-4} \mathbf{l}_{-2}+\frac{56-52 \Delta-2 c+4 c \Delta}{5(\Delta+2)} \mathbf{l}_{-6}, \\
\phi_{3,3}^{\text {even }} \cong & \mathbf{l}_{-2}^{3}+\frac{6+3 c-76 \Delta+4 c \Delta}{6(\Delta+2)} \mathbf{l}_{-2} \mathbf{l}_{-4} \\
& +\frac{-6544+498 c-5 c^{2}+2152 \Delta-314 c \Delta+10 c^{2} \Delta-448 \Delta^{2}+16 c \Delta^{2}}{60(\Delta+2)} \mathbf{l}_{-6},
\end{aligned}
$$

where for simplicity we took $\Delta \equiv \Delta_{\alpha}$. We hope it will not cause some confusion by mixing this $\Delta$ with the anisotropy parameter of the XXZ model. In the above formula (2.21) we imply

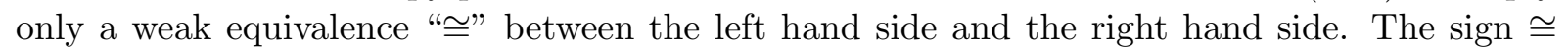
means that the left and right hand sides being substituted into the corresponding expectation value give the same result by acting on $\Phi_{\alpha}(0)$ and $\phi_{\alpha}(0)$ respectively. In other words $A \cong B$ if and only if

$$
Z_{R}^{\kappa, \kappa}\left(A\left(\Phi_{\alpha}(0)\right)\right)=\frac{\left\langle\Delta_{-}\left|B\left(\phi_{\alpha}(0)\right)\right| \Delta_{+}\right\rangle}{\left\langle\Delta_{-}\left|\phi_{\alpha}(0)\right| \Delta_{+}\right\rangle}
$$

with the functional $Z_{R}^{\kappa, \kappa^{\prime}}$ defined in (2.17). In the next sections we will generalize the scheme of [4] to the case of excited states. 


\section{TBA for excited states}

In fact, the Jimbo, Miwa, Smirnov theorem (2.7) is valid not only for eigenvectors $|\kappa\rangle,|\kappa+\alpha-s, s\rangle$ associated with maximal eigenvalues in the corresponding sectors, but also for eigenvectors corresponding to excited states. Here we consider only special excited states, namely, the socalled particle-hole excitations [17]. Some motivation for this is as follows. In [7] Bazhanov, Lukyanov and Zamolodchikov formulated several assumptions about the analytical properties of the eigenvalues of the $Q$-operator with respect to the square of the spectral parameter $\zeta^{2}$, in particular, that its zeroes in the complex $\zeta^{2}$-plane are either real or occur in complex conjugated pairs, that an infinite number of zeroes are real and positive and that there may be only a finite number of complex or real negative zeroes. An important assumption is that the real zeroes accumulate towards $+\infty$ in the variable $\zeta^{2}$. Further, in [18] the authors proposed a conjecture that for the asymptotic analysis, when the parameter $\kappa$ becomes large, only real positive zeroes corresponding either to the vacuum or to the excited states are important. The question why real negative zeroes or complex zeroes are not important for the asymptotic analysis seems hard. Usually one uses experience coming from the numerical study and also the arguments based on the analysis of a small vicinity of the free-fermion point $\nu=1 / 2$. Counting arguments play important role here as well. But we do not know any rigorous proof of this statement. Any further discussion of this subtle question would lead us beyond the scope of this paper.

Let us start with the case of a lattice with an even finite number of sites $\mathbf{n}$ in the Matsubara direction. The Bethe ansatz equations (BAE) are usually deduced from the Baxter's TQ-relation [9] for the eigenvalues $T(\zeta, \kappa, s), Q(\zeta, \kappa, s)$ of the transfer matrix $T_{\mathbf{M}}(\zeta, \kappa)$ and the $Q$-operator $Q_{\mathbf{M}}(\zeta, \kappa)$ defined by the formula (3.3) of [4]

$$
T(\zeta, \kappa, s) Q(\zeta, \kappa, s)=d(\zeta) Q(q \zeta, \kappa, s)+a(\zeta) Q\left(q^{-1} \zeta, \kappa, s\right), \quad Q(\zeta, \kappa, s)=\zeta^{-\kappa+s} A(\zeta, \kappa, s)
$$

with polynomial dependence of the functions $T$ and $A$ on the spectral parameter $\zeta$ in every spin sector $s, 0 \leq s \leq \frac{\mathbf{n}}{2}$ and

$$
a(\zeta)=\left(1-q \zeta^{2}\right)^{\mathbf{n}}, \quad d(\zeta)=\left(1-q^{-1} \zeta^{2}\right)^{\mathbf{n}} .
$$

In the TBA approach one introduces auxiliary function

$$
\mathfrak{a}(\zeta, \kappa, s)=\frac{d(\zeta) Q(q \zeta, \kappa, s)}{a(\zeta) Q\left(q^{-1} \zeta, \kappa, s\right)}
$$

which satisfies the BAE

$$
\mathfrak{a}\left(\xi_{j}, \kappa, s\right)=-1, \quad j=1, \ldots, \frac{\mathbf{n}}{2}-s
$$

with $\frac{\mathbf{n}}{2}-s$ zeros $\xi_{j}$ of $Q(\zeta, \kappa, s)$ called Bethe roots. The BAE in the logarithmic form look

$$
\log \mathfrak{a}\left(\xi_{j}, \kappa, s\right)=\pi i m_{j}
$$

where $m_{j}$ are pairwise non-coinciding odd integers. The ground state corresponds to $s=0$ and the choice $m_{j}=2 j-1$. The following picture describes this situation

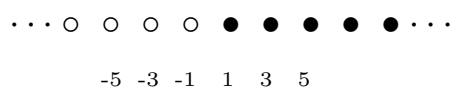

where the black circles correspond to "particles" and have positive odd coordinates $m_{j}$ while the white circles correspond to "holes" and have negative coordinates. The particle-hole excitations can be got when some of "particles" are moved to positions with negative coordinates. Let us denote $I^{(+, k)}$ an ordered subset of positive coordinates $I_{1}^{(+, k)}<\cdots<I_{k}^{(+, k)} \leq \mathbf{n}$ which 
correspond to the positions of created holes and $I^{(-, k)}$ correspond to negative coordinates of the moved particles ${ }^{6}-I_{k}^{(-, k)}<\cdots<-I_{1}^{(-, k)}$ :

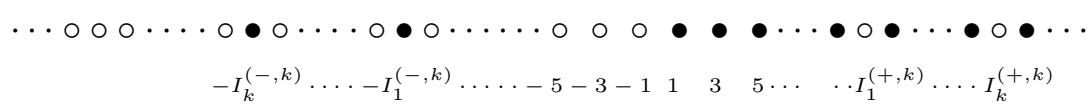

We will denote the corresponding vector and co-vector $\left|\kappa ; I^{(+, k)}, I^{(-, k)}\right\rangle$ and $\left\langle\kappa ; I^{(+, k)}, I^{(-, k)}\right|$. Let $\xi_{r}^{-}, r=1, \ldots, k$ be the Bethe roots corresponding to the particles moved into the positions with negative coordinates $-I_{r}^{(-, k)}$ and $\xi_{r}^{+}$correspond to the holes with positive positions $I_{r}^{(+, k)}$.

Following the papers $[5,7,19,20]$, we can rewrite the BAE (3.1) in form of the non-linear integral equation

$$
\log \mathfrak{a}(\zeta, \kappa, s)=-2 \pi i \nu(\kappa-s)+\log \left(\frac{d(\zeta)}{a(\zeta)}\right)-\int_{\gamma_{(s, k)}} K(\zeta / \xi) \log (1+\mathfrak{a}(\xi, \kappa, s)) \frac{d \xi^{2}}{\xi^{2}},
$$

where the contour $\gamma_{(s, k)}$ goes around all the Bethe roots $\xi_{j}$ including the moved ones $\xi_{j_{r}}=\xi_{r}^{-}$ in the clockwise direction and the kernel

$$
K(\zeta, \alpha)=\frac{1}{2 \pi i} \Delta_{\zeta} \psi(\zeta, \alpha), \quad K(\zeta)=K(\zeta, 0), \quad \Delta_{\zeta} f(\zeta)=f(q \zeta)-f\left(q^{-1} \zeta\right)
$$

For the case of the ground state $s=0, k=0$ we have $\gamma_{(0,0)}=\gamma$ with the contour $\gamma$ used in Section 3 of [4]. Let us for simplicity stay with the case $s=0$. One can rewrite (3.3) replacing the contour $\gamma_{(0, k)}$ by $\gamma$ and taking into account the contribution of the residues from the moved Bethe roots $\xi_{r}^{-}$and holes $\xi_{r}^{+}$. There are also other equations coming from (3.2). Altogether we have the following set of equations

$$
\begin{aligned}
\log \mathfrak{a}(\zeta, \kappa)= & -2 \pi i \nu \kappa+\log \left(\frac{d(\zeta)}{a(\zeta)}\right)+\sum_{r=1}^{k}\left(g\left(\zeta / \xi_{r}^{+}\right)-g\left(\zeta / \xi_{r}^{-}\right)\right) \\
& -\int_{\gamma} K(\zeta / \xi) \log (1+\mathfrak{a}(\xi, \kappa)) \frac{d \xi^{2}}{\xi^{2}}, \\
\log \mathfrak{a}\left(\xi_{r}^{ \pm}, \kappa\right)= & \mp \pi i I_{r}^{( \pm, k)}, \quad r=1, \ldots, k,
\end{aligned}
$$

where $g(\zeta)=\log \frac{1-q^{2} \zeta^{2}}{1-q^{-2} \zeta^{2}}$.

Now we can consider the scaling limit and generalize the analysis of Section 10 of [4]. Let us start with a small remark. So far, we considered only particles which "jump" to the left. In principle, for the case of finite $\mathbf{n}$ we should also consider particles which "jump" to the right. But in the scaling limit when $\mathbf{n} \rightarrow \infty$ it is implied that the right tail of the Bethe ansatz phases in the ground state is infinite. So, the jumps to the right are irrelevant in the scaling limit. Therefore we will not discuss them here. Let us introduce the functions

$$
\begin{aligned}
& T^{\mathrm{sc}}(\lambda, \kappa)=\lim _{\mathbf{n} \rightarrow \infty,}, a \rightarrow 0,2 \pi R=\mathbf{n} a \\
& Q^{\mathrm{sc}}(\lambda, \kappa)=\lim _{\mathbf{n} \rightarrow \infty,} \lim _{a \rightarrow 0,2 \pi R=\mathbf{n} a} \bar{a}^{\nu \kappa} Q\left(\lambda \bar{a}^{\nu}, \kappa\right)
\end{aligned}
$$

and taking into account that for $1 / 2<\nu<1$ the ratio $a(\zeta) / d(\zeta) \rightarrow 1$ in the scaling limit with $\zeta$ related to $\lambda$ as in (2.11), we get the scaling limit of the auxiliary function $\mathfrak{a}$ as

$$
\mathfrak{a}^{\mathrm{sc}}(\lambda, \kappa)=\frac{Q^{\mathrm{sc}}(\lambda q, \kappa)}{Q^{\mathrm{sc}}\left(\lambda q^{-1}, \kappa\right)}
$$

\footnotetext{
${ }^{6}$ The case of $s>0$ can be treated similarly to the case $s=0$. The only difference is that for the finite $\mathbf{n}$ case one has less Bethe roots.
} 
We would like to study the asymptotic behavior of $\mathfrak{a}^{\text {sc }}$ for $\lambda^{2} \rightarrow \infty$ and $\kappa \rightarrow \infty$ in such a way that the variable

$$
t=c(\nu)^{-1} \frac{\lambda^{2}}{\kappa^{2 \nu}}
$$

is kept fixed with

$$
c(\nu)=\Gamma(\nu)^{-2} e^{\delta}\left(\frac{\nu}{2 R}\right)^{2 \nu}, \quad \delta=-\nu \log \nu-(1-\nu) \log (1-\nu) .
$$

Then the function

$$
F(t, \kappa):=\log \mathfrak{a}^{\mathrm{sc}}(\lambda, \kappa)
$$

satisfies the equation

$$
\begin{aligned}
F(t, \kappa) & -\int_{1}^{\infty} K(t / u) F(u, \kappa) \frac{d u}{u}=-2 \pi i \nu \kappa+\sum_{r=1}^{k}\left(g\left(t / t_{r}^{+}\right)-g\left(t / t_{r}^{-}\right)\right) \\
& -\int_{1}^{e^{i \epsilon} \infty} K(t / u) \log \left(1+e^{F(u, \kappa)}\right) \frac{d u}{u}+\int_{1}^{e^{-i \epsilon} \infty} K(t / u) \log \left(1+e^{-F(u, \kappa)}\right) \frac{d u}{u},
\end{aligned}
$$

where $\epsilon$ is a small positive number and

$$
t_{r}^{ \pm}=c(\nu)^{-1} \frac{\xi_{r}^{ \pm 2}}{(\kappa \bar{a})^{2 \nu}}
$$

With a slight abuse of notation we will write

$$
K(t)=\frac{1}{2 \pi i} \cdot \frac{1}{2}\left(\frac{t q^{2}+1}{t q^{2}-1}-\frac{t q^{-2}+1}{t q^{-2}-1}\right), \quad g(t)=\log \frac{t q^{2}-1}{t q^{-2}-1} .
$$

Introducing the resolvent $R(t, u)$

$$
R(t, u)-\int_{1}^{\infty} \frac{d v}{v} R(t, v) K(v / u)=K(t / u) \quad(t, u>1)
$$

and

$$
\begin{aligned}
& G(t ; u, v)=G(t, u)-G(t, v), \\
& G(t, u)=((I+R) g)(t, u)=g(t / u)+\int_{1}^{\infty} R(t, v) g(v / u) \frac{d v}{v},
\end{aligned}
$$

one can rewrite the equation (3.6) as follows

$$
\begin{aligned}
F(t, \kappa)= & \kappa F_{0}(t)+\sum_{r=1}^{k} G\left(t ; t_{r}^{+}, t_{r}^{-}\right) \\
& -\int_{1}^{e^{i \epsilon} \infty} R(t, u) \log \left(1+e^{F(u, \kappa)}\right) \frac{d u}{u}+\int_{1}^{e^{-i \epsilon} \infty} R(t, u) \log \left(1+e^{-F(u, \kappa)}\right) \frac{d u}{u},
\end{aligned}
$$

where $t_{r}^{ \pm}$depend on $\kappa$ and can be defined from the equations

$$
F\left(t_{r}^{ \pm}, \kappa\right)=\mp \pi i I_{r}^{( \pm, k)}, \quad r=1, \ldots, k
$$


and $F_{0}$ is the same as in [4]. It satisfies the integral equation

$$
\left((I-K) F_{0}\right)(t)=-2 \pi i \nu
$$

where as in (3.7), the contraction is defined on the interval $[1, \infty)$

$$
K f(t)=\int_{1}^{\infty} K(t / u) f(u) \frac{d u}{u} .
$$

It follows from the WKB technique [7] that the asymptotic behavior at $t \rightarrow \infty$

$$
F_{0}(t)=\text { const } \cdot t^{\frac{1}{2 \nu}}+O\left(t^{-\frac{1}{2 \nu}}\right) \text {. }
$$

Then the equation (3.10) can be uniquely solved by the Wiener-Hopf factorization technique

$$
F_{0}(t)=\int_{\mathbb{R}-\frac{i}{2 \nu}-i 0} d l t^{i l} S(l) \frac{-i f}{l\left(l+\frac{i}{2 \nu}\right)} \quad(t>1),
$$

where

$$
f=\frac{1}{2 \sqrt{2(1-\nu)}}
$$

and the function

$$
S(k)=\frac{\Gamma(1+(1-\nu) i k) \Gamma(1 / 2+i \nu k)}{\Gamma(1+i k) \sqrt{2 \pi(1-\nu)}} e^{i \delta k}
$$

with $\delta$ defined in (3.5) satisfies factorization condition

$$
1-\hat{K}(k)=S(k)^{-1} S(-k)^{-1}, \quad \hat{K}(k)=\frac{\sinh (2 \nu-1) \pi k}{\sinh \pi k},
$$

where $\hat{K}$ stands for the Mellin transform of the kernel $K$

$$
\hat{K}(k)=\int_{0}^{\infty} K(t) t^{-i k} \frac{d t}{t}, \quad K(t)=\frac{1}{2 \pi} \int_{-\infty}^{\infty} \hat{K}(k) t^{i k} d k .
$$

Below we also use the asymptotic expansion of $S(k)$ at $k \rightarrow \infty$

$$
S(k) \simeq 1+\sum_{j=1}^{\infty} S_{j}(i k)^{-j} .
$$

For example, the two leading terms look

$$
S_{1}=\frac{(1+\nu)(2 \nu-1)}{24(1-\nu) \nu}, \quad S_{2}=\frac{(1+\nu)^{2}(2 \nu-1)^{2}}{1152(1-\nu)^{2} \nu^{2}} .
$$

The same method leads to

$$
\begin{aligned}
R(t, u) & =\int_{-\infty}^{\infty} \frac{d l}{2 \pi} \int_{-\infty}^{\infty} \frac{d m}{2 \pi} t^{i l} u^{i m} S(l) S(m) \hat{K}(m) \frac{-i}{l+m-i 0} \\
& =K(t / u)+\int_{-\infty}^{\infty} \frac{d l}{2 \pi} \int_{-\infty}^{\infty} \frac{d m}{2 \pi} t^{i l} u^{i m} S(l) S(m) \hat{K}(l) \hat{K}(m) \frac{-i}{l+m-i 0} .
\end{aligned}
$$


One can solve the equation (3.8) iteratively using the asymptotic expansion

$$
F(t, \kappa)=\sum_{n=0}^{\infty} \kappa^{-n+1} F_{n}(t)
$$

An important difference of this expansion with the formula (10.3) of [4] is that here also even degrees of $\kappa$ may appear. Since

$$
-\frac{1}{2 \pi i} u \frac{\partial}{\partial u} g(t / u)=K(t / u)
$$

we can get the following equation by differentiating (3.7)

$$
-\frac{1}{2 \pi i} u \frac{\partial}{\partial u} G(t / u)=((I+R) K)(t, u)=R(t, u)
$$

and then using (3.13)

$$
G(t, u)=-2 \pi i \int_{-\infty}^{\infty} \frac{d l}{2 \pi} \int_{-\infty}^{\infty} \frac{d m}{2 \pi} t^{i l} u^{i m} S(l) \hat{K}(l) S(m) \frac{-i}{l+m-i 0} \frac{-i}{m+i 0},
$$

where we used the regularization $m+i 0$ in such a way that $G(t, u) \rightarrow 0$ for $u \rightarrow \infty$ but in fact, this does not matter because $G(t, u)$ enters into the equation (3.8) only through the difference $G(t, u)-G(t, v)$.

One introduces the function $\Psi(l, \kappa)$ which has an asymptotic expansion

$$
\Psi(l, \kappa) \simeq \sum_{n=0}^{\infty} \kappa^{-n+1} \Psi_{n}(l), \quad \Psi_{0}(l)=-\frac{i f}{l\left(l+\frac{i}{2 \nu}\right)}
$$

related to the function $F(t, \kappa)$ via

$$
F(t, \kappa)=\kappa F_{0}(t)+\int_{-\infty}^{\infty} d l t^{i l} S(l) \hat{K}(l)\left(\Psi(l, \kappa)-\kappa \Psi_{0}(l)\right) .
$$

It is convenient to introduce

$$
p:=f \kappa,
$$

where $f$ is defined in (3.11) and consider the asymptotic expansion with respect to $p$ instead of $\kappa$.

Using (3.15), one can rewrite (3.6) in the following equivalent form

$$
\begin{aligned}
\Psi^{(p)}(l, p)-p \Psi_{0}^{(p)}(l)= & \sum_{r=1}^{k} \hat{G}\left(l ; t_{r}^{+}, t_{r}^{-}\right)-\frac{i}{p}\left\{\int_{0}^{-i \infty+\epsilon} \frac{d x}{2 \pi} \hat{R}\left(l, e^{i x / p}\right) \log \left(1+e^{F^{(p)}\left(e^{i x / p}, p\right)}\right)\right. \\
& \left.+\int_{0}^{i \infty+\epsilon} \frac{d x}{2 \pi} \hat{R}\left(l, e^{-i x / p}\right) \log \left(1+e^{-F^{(p)}\left(e^{-i x / p}, p\right)}\right)\right\}
\end{aligned}
$$

where $\epsilon$ is a small positive number and by definition

$$
F^{(p)}(t, p):=F(t, \kappa), \quad \Psi^{(p)}(l, p):=\Psi(l, \kappa), \quad \Psi_{0}^{(p)}(l):=\frac{1}{f} \Psi_{0}(l)=-\frac{i}{l\left(l+\frac{i}{2 \nu}\right)}
$$

and then

$$
\hat{R}\left(l, e^{i x / p}\right)=\operatorname{res}_{h}\left[\frac{e^{-h x / p}}{l+h} S(h)\right], \quad \hat{G}(l ; y, z)=-\operatorname{res}_{h}\left[\frac{e^{-h y / p}-e^{-h z / p}}{h(l+h)} S(h)\right],
$$


where $\operatorname{res}_{h}$ is the coefficient at $h^{-1}$ in the expansion at $h=\infty$. Taking

$$
F^{(p)}\left(e^{i x / p}, p\right)=-2 \pi(x-\bar{F}(x, p)),
$$

we obtain the Taylor series at $x=0$

$$
\bar{F}(x, p)=x+\operatorname{res}_{h}\left[e^{-h x / p} S(h) i \Psi^{(p)}(h, p)\right] .
$$

As the asymptotic series with respect to $p$ at $p \rightarrow \infty$, it starts with $p^{-1}$. The $2 k$ parameters $t_{r}^{ \pm}$ participating in (3.9) and (3.16) are functions of $\kappa$ or equivalently of $p$. We take them in the following form

$$
t_{r}^{ \pm}(\kappa)=e^{\frac{i x_{r}^{ \pm}(p)}{p}}, \quad x_{r}^{ \pm}(p)=\sum_{j=0}^{\infty} x_{r, j}^{ \pm} p^{-j} .
$$

Then we get from (3.16)

$$
\Psi^{(p)}(l, p)=\sum_{r=1}^{k} \hat{G}\left(l ; x_{r}^{+}(p), x_{r}^{-}(p)\right)-\frac{i p}{l\left(l+\frac{i}{2 \nu}\right)}+H(l, p),
$$

where

$$
\begin{aligned}
H(l, p)= & -\frac{2 i}{p} \int_{0}^{\infty} \frac{d x}{2 \pi}\left\{\operatorname{res}_{h}\left(\frac{e^{-h x / p}}{l+h} S(h)\right)\right. \\
& \left.\times \sum_{n=0}^{\infty} \frac{1}{n !} \bar{F}(x, p)^{n}\left(-\frac{\partial}{\partial x}\right)^{n}\right\}_{\text {even }} \log \left(1+e^{-2 \pi x}\right) .
\end{aligned}
$$

Here $\left\{f\left(x, \frac{\partial}{\partial x}\right)\right\}_{\text {even }}=\frac{1}{2}\left(f\left(x, \frac{\partial}{\partial x}\right)+f\left(-x,-\frac{\partial}{\partial x}\right)\right)$. The parameters $x_{r}^{ \pm}(p)$ are to be determined from the condition

$$
\bar{F}\left(x_{r}^{ \pm}(p), p\right)=x_{r}^{ \pm}(p) \mp \frac{i}{2} I_{r}^{( \pm, k)}
$$

which is $O\left(p^{-1}\right)$ if

$$
x_{r, 0}^{ \pm}= \pm \frac{i}{2} I_{r}^{( \pm, k)}
$$

So, we come to the iterative scheme which allows us to compute $F^{(p)}(t, p)$ to any order of $p^{-1}$. One can see that the following expansions are consistent with the above equations:

$$
\begin{aligned}
& \bar{F}(x, p)=\sum_{j=0}^{\infty} \bar{F}_{j}(x) p^{-j-1}, \\
& H(l, p)=\sum_{j=0}^{\infty} H_{j}(l) p^{-j-1}, \\
& \hat{G}(l ; y, z)=\sum_{j=0}^{\infty} \hat{G}_{j}(l ; y, z) p^{-j-1}
\end{aligned}
$$

and in agreement with (3.14)

$$
\Psi^{(p)}(l, p)=\sum_{j=0}^{\infty} \Psi_{j}^{(p)}(l) p^{-j+1} .
$$


Let us explain the very first iteration step. We first compute $H_{0}$ in the expansion (3.24) using (3.20), the formula

$$
\int_{0}^{\infty} \frac{d x}{2 \pi} x^{m}\left(-\frac{\partial}{\partial x}\right)^{n} \log \left(1+e^{-2 \pi x}\right)=m !\left(1-2^{-m-1+n}\right) \frac{\zeta(m-n+2)}{(2 \pi)^{m-n+2}}
$$

and the fact that only the term with $n=0$ in the sum at the right hand side of (3.20) contributes because $\bar{F}$ is of order $p^{-1}$. So, we easily come to the result

$$
H_{0}(l)=-\frac{i}{24}
$$

Then we substitute it into (3.19) and calculate the leading order of the function $\hat{G}$ taking into account the condition (3.22). As a result we obtain a few leading orders of $\Psi^{(p)}$ :

$$
\Psi^{(p)}(l, p)=-\frac{i p}{l\left(l+\frac{i}{2 \nu}\right)}+\left(\sum_{r=1}^{k} \frac{i}{2}\left(I_{r}^{(+, k)}+I_{r}^{(-, k)}\right)-\frac{i}{24}\right) p^{-1}+O\left(p^{-2}\right) .
$$

This we substitute into the formula (3.17) and get $\bar{F}_{0}$ from $(3.23)$

$$
\bar{F}_{0}(x)=-\left(\frac{i}{4 \nu}+\frac{i S_{1}}{2}\right) x^{2}+S_{1}\left(\sum_{r=1}^{k} \frac{i}{2}\left(I_{r}^{(+, k)}+I_{r}^{(-, k)}\right)-\frac{i}{24}\right),
$$

where $S_{1}$ is given by (3.12). Then we take the equation (3.21) up to the order $p^{-1}$ and easily solve it

$$
x_{s, 1}^{ \pm}=\left(\frac{i}{4 \nu}+\frac{i S_{1}}{2}\right)\left(\frac{I_{s}^{( \pm, k)}}{2}\right)^{2}+S_{1}\left(\sum_{r=1}^{k} \frac{i}{2}\left(I_{r}^{(+, k)}+I_{r}^{(-, k)}\right)-\frac{i}{24}\right) .
$$

One can do the second iteration by repeating this procedure. In Appendices A and B we show the result of such a calculation for few next orders with respect to $p^{-1}$.

\section{The function $\omega^{\mathrm{sc}}$}

Also we need to generalize the expressions (11.5), (11.6) of [4] for the function $\omega^{\mathrm{sc}}$ to the case of excited states. Still we take the condition $\kappa=\kappa^{\prime}$ for which we can choose the excited state for the spin 0 sector and for the sector with spin $s$ in such a way that $\rho\left(\lambda \mid \kappa, \kappa^{\prime}\right)=1$. The reasoning here is quite similar to that one described in Section 4 of [4]. Actually, one can start with the similar expression to (11.1) of [4] but with a generalized dressed resolvent $R_{\text {dress }}$

$$
\omega^{\mathrm{sc}}(\lambda, \mu \mid \kappa, \kappa, \alpha)=\left(f_{\text {left }} \star_{k} f_{\text {right }}+f_{\text {left }} \star_{k} R_{\text {dress }} \star_{k} f_{\text {right }}\right)(\lambda, \mu)+\omega_{0}(\lambda, \mu \mid \alpha),
$$

where $^{7}$

$$
\begin{aligned}
& f_{\text {left }}(\lambda, \mu, \alpha)=\frac{1}{2 \pi i} \delta_{\lambda}^{-} \psi_{0}(\lambda / \mu, \alpha), \quad f_{\text {right }}(\lambda, \mu, \alpha)=\delta_{\mu}^{-} \psi_{0}(\lambda / \mu, \alpha), \\
& \delta_{\lambda}^{-} f(\lambda)=f(q \lambda)-f(\lambda), \quad \omega_{0}(\lambda, \mu \mid \alpha)=\delta_{\lambda}^{-} \delta_{\mu}^{-} \Delta_{\lambda}^{-1} \psi_{0}(\lambda / \mu, \alpha), \\
& \psi_{0}(\lambda, \alpha)=\frac{\lambda^{\alpha}}{\lambda^{2}-1}, \quad \Delta_{\lambda} f(\lambda)=f(q \lambda)-f\left(q^{-1} \lambda\right)
\end{aligned}
$$

\footnotetext{
${ }^{7}$ In comparison with (11.1) of [4] we take instead of the function $\psi$ defined in (2.5) the function $\psi_{0}$. The result does not change if we also change the kernel $K_{\alpha} \rightarrow K_{\alpha, 0}$ as it is done in (4.2).
} 
with

$$
\Delta_{\lambda}^{-1} \psi_{0}(\lambda, \alpha)=-V P \int_{0}^{\infty} \frac{\psi_{0}(\mu, \alpha)}{2 \nu\left(1+(\lambda / \mu)^{\frac{1}{\nu}}\right)} \frac{d \mu^{2}}{2 \pi i \mu^{2}},
$$

where the principal value is taken for the pole $\mu^{2}=1$. The contraction $\star_{k}$ means

$$
f \star_{k} g=\int_{\tilde{\gamma}_{(0, k)}} f(\lambda) g(\lambda) d m(\lambda), \quad d m(\lambda)=\frac{d \lambda^{2}}{\lambda^{2}\left(1+\mathfrak{a}^{\mathrm{sc}}(\lambda, \kappa)\right)}
$$

with the contour $\tilde{\gamma}_{(0, k)}$ which corresponds to $\gamma_{(0, k)}$ from the equation (3.3) but taken for variable $\lambda^{2}$ instead of $\zeta^{2}=\lambda^{2} \bar{a}^{2 \nu}$. We remind the reader that the contour $\gamma_{(0, k)}$ was taken around all the Bethe roots in the clockwise direction in case of the excited state with $k$ particles and $k$ holes for the zero spin sector.

The resolvent $R_{\text {dress }}$ fulfills the integral equation

$$
R_{\text {dress }}-R_{\text {dress }} \star_{k} K_{\alpha, 0}=K_{\alpha, 0}, \quad K_{\alpha, 0}(\lambda)=\Delta_{\lambda} \psi_{0}(\lambda, \alpha) .
$$

Applying a similar trick which we used to derive (3.4), namely, deforming the contour $\tilde{\gamma}_{(0, k)} \rightarrow \tilde{\gamma}_{(0,0)}$ and taking into account additional terms coming from the residues corresponding to particles and holes, we can obtain

$$
\begin{aligned}
& R_{\mathrm{dress}}(t, u)-R(t, u, \alpha)=2 \pi i \sum_{r=1}^{k}\left(\frac{R\left(t, t_{r}^{+}, \alpha\right) R_{\mathrm{dress}}\left(t_{r}^{+}, u\right)}{F^{\prime}\left(t_{r}^{+}, \kappa\right)}-\frac{R\left(t, t_{r}^{-}, \alpha\right) R_{\mathrm{dress}}\left(t_{r}^{-}, u\right)}{F^{\prime}\left(t_{r}^{-}, \kappa\right)}\right) \\
& \quad-\int_{1}^{e^{i \epsilon} \infty} \frac{R(t, v ; \alpha) R_{\mathrm{dress}}(v, u)}{1+e^{-F(v, \kappa)}} \frac{d v}{v}-\int_{1}^{e^{-i \epsilon} \infty} \frac{R(t, v ; \alpha) R_{\mathrm{dress}}(v, u)}{1+e^{F(v, \kappa)}} \frac{d v}{v} \\
& F^{\prime}(t, \kappa):=t \frac{\partial}{\partial t} F(t, \kappa),
\end{aligned}
$$

where as in [4], we introduced the "undressed" resolvent $R(t, u, \alpha)$ which satisfies the equation

$$
R(t, u, \alpha)-\int_{1}^{\infty} \frac{d v}{v} R(v, u, \alpha) K_{0}(t / v, \alpha)=K_{0}(t / u, \alpha)
$$

with the kernel

$$
K_{0}(t, \alpha)=\frac{1}{2 \pi i}\left(\frac{\left(t q^{2}\right)^{\alpha / 2}}{t q^{2}-1}-\frac{\left(t q^{-2}\right)^{\alpha / 2}}{t q^{-2}-1}\right)
$$

corresponding to the above kernel $K_{\alpha, 0}$. The solution of (4.4) again can be got by the WienerHopf factorization technique

$$
\begin{aligned}
R(t, u, \alpha)= & K_{0}(t / u, \alpha) \\
& +\int_{-\infty}^{\infty} \frac{d l}{2 \pi} \int_{-\infty}^{\infty} \frac{d m}{2 \pi} t^{i l} u^{i m} S(l, \alpha) S(m, 2-\alpha) \hat{K}(l, \alpha) \hat{K}(m, 2-\alpha) \frac{-i}{l+m-i 0}
\end{aligned}
$$

with the Mellin-transform

$$
\hat{K}(k, \alpha)=\frac{\sinh \pi\left((2 \nu-1) k-\frac{i \alpha}{2}\right)}{\sinh \pi\left(k+\frac{i \alpha}{2}\right)}
$$

corresponding to the kernel $K_{0}(t, \alpha)$ and

$$
S(k, \alpha)=\frac{\Gamma\left(1+(1-\nu) i k-\frac{\alpha}{2}\right) \Gamma\left(\frac{1}{2}+i \nu k\right)}{\Gamma\left(1+i k-\frac{\alpha}{2}\right) \sqrt{2 \pi}(1-\nu)^{(1-\alpha) / 2}} e^{i \delta k},
$$




$$
1-\hat{K}(k, \alpha)=S(k, \alpha)^{-1} S(-k, 2-\alpha)^{-1} .
$$

We assume that

$$
0<\alpha<2 \text {. }
$$

Now we take the ansatz for $R_{\text {dress }}(t, u)$

$$
\begin{aligned}
R_{\mathrm{dress}}(t, u)= & K_{0}(t / u, \alpha) \\
& +\int_{-\infty}^{\infty} \frac{d l}{2 \pi} \int_{-\infty}^{\infty} \frac{d m}{2 \pi} t^{i l} u^{i m} S(l, \alpha) S(m, 2-\alpha) \hat{K}(l, \alpha) \hat{K}(m, 2-\alpha) \Theta(l, m \mid p, \alpha)
\end{aligned}
$$

with the asymptotic expansion ${ }^{8}$ at $p \rightarrow \infty$

$$
\Theta(l, m \mid p, \alpha) \simeq \sum_{n=0}^{\infty} \Theta_{n}(l, m \mid \alpha) p^{-n}, \quad \Theta_{0}(l, m \mid \alpha)=-\frac{i}{l+m}
$$

and substitute it into the equation (4.3). As a result we get the equation which allows us to calculate all $\Theta_{n}$ by iterations

$$
\begin{aligned}
& \Theta(l, m \mid p, \alpha)-\Theta_{0}(l, m \mid \alpha)=\frac{1}{p} \operatorname{res}_{l^{\prime}} \operatorname{res}_{m^{\prime}}\left[S\left(l^{\prime}, 2-\alpha\right) S\left(m^{\prime}, \alpha\right) \Theta\left(m^{\prime}, m \mid p, \alpha\right) /\left(l+l^{\prime}\right)\right. \\
& \times\left(-i \sum_{r=1}^{k} e^{-\left(l^{\prime}+m^{\prime}\right) x_{r}^{+}(p) / p} /\left(\bar{F}^{\prime}\left(x_{r}^{+}(p), p\right)-1\right)+i \sum_{r=1}^{k} e^{-\left(l^{\prime}+m^{\prime}\right) x_{r}^{-}(p) / p} /\left(\bar{F}^{\prime}\left(x_{r}^{-}(p), p\right)-1\right)\right. \\
& \left.\left.+2 \sum_{n=0}^{\infty} \frac{1}{n !} \int_{0}^{\infty} d x\left\{e^{-\left(l^{\prime}+m^{\prime}\right) x / p} \bar{F}(x, p)^{n}\left(-\frac{\partial}{\partial x}\right)^{n}\right\}_{\text {odd }} \frac{1}{1+e^{2 \pi x}}\right)\right],
\end{aligned}
$$

where the odd part $\left\{f\left(x, \frac{\partial}{\partial x}\right)\right\}_{\text {odd }}=\frac{1}{2}\left(f\left(x, \frac{\partial}{\partial x}\right)-f\left(-x,-\frac{\partial}{\partial x}\right)\right)$ and

$$
\bar{F}^{\prime}(x, p):=\frac{\partial}{\partial x} \bar{F}(x, p) \text {. }
$$

Performing iterations implies that enough many orders in the expansion of $\bar{F}(x, p)$ and $x^{ \pm}(p)$ with respect to $p^{-1}$ were obtained by means of the iteration scheme described in the previous section. For a few leading terms in the expansion for $\Theta(l, m \mid p, \alpha)$ we get

$$
\begin{aligned}
\Theta(l, m \mid p, \alpha)= & -\frac{i}{l+m}+\left(\frac{1}{24 \nu}-\frac{1}{2 \nu} \sum_{r=1}^{k}\left(I_{r}^{(+, k)}+I_{r}^{(-, k)}\right)\right) \\
& \times\left(-i \nu(l+m)-\frac{1}{2}+\Delta_{\alpha}\right) p^{-2}+O\left(p^{-3}\right)
\end{aligned}
$$

where $\Delta_{\alpha}$ is given by (2.2). Some other terms of this expansion will be shown in Appendix $\mathrm{C}$ for the case $k=1$.

One can derive relation like (11.5) of [4] using the form (4.1), integral equations (4.2), (4.3) for the dressed resolvent $R_{\text {dress }}$ and the ansatz (4.5)

$$
\omega^{\mathrm{sc}}(\lambda, \mu \mid \kappa, \kappa, \alpha)
$$

\footnotetext{
${ }^{8}$ Here $\Theta_{n}(l, m \mid \alpha)$ are different from those introduced in [4] since we should take into account the contribution of terms with odd degrees $n$ also.
} 


$$
\simeq \frac{1}{2 \pi i} \int_{-\infty}^{\infty} d l \int_{-\infty}^{\infty} d m \tilde{S}(l, \alpha) \tilde{S}(m, 2-\alpha) \Theta(l+i 0, m \mid p, \alpha)\left(\frac{e^{\delta+\pi i \nu} \lambda^{2}}{\kappa^{2 \nu} c(\nu)}\right)^{i l}\left(\frac{e^{\delta+\pi i \nu} \mu^{2}}{\kappa^{2 \nu} c(\nu)}\right)^{i m}
$$

where we returned to the variables $\lambda, \mu$ and

$$
\tilde{S}(k, \alpha)=\frac{\Gamma\left(-i k+\frac{\alpha}{2}\right) \Gamma\left(\frac{1}{2}+i \nu k\right)}{\Gamma\left(-i(1-\nu) k+\frac{\alpha}{2}\right) \sqrt{2 \pi}(1-\nu)^{(1-\alpha) / 2}} .
$$

The asymptotic expansion at $\lambda, \mu \rightarrow \infty$ can be obtained by computing the residues of the functions $\tilde{S}(l, \alpha)$ and $\tilde{S}(m, 2-\alpha)$

$$
\omega^{\mathrm{sc}}(\lambda, \mu \mid \kappa, \kappa, \alpha) \simeq \sum_{r, s=1}^{\infty} \frac{1}{r+s-1} D_{2 r-1}(\alpha) D_{2 s-1}(2-\alpha) \lambda^{-\frac{2 r-1}{\nu}} \mu^{-\frac{2 s-1}{\nu}} \Omega_{2 r-1,2 s-1}(p, \alpha),
$$

where

$$
D_{2 n-1}(\alpha)=\frac{1}{\sqrt{i \nu}} \Gamma(\nu)^{-\frac{2 n-1}{\nu}}(1-\nu)^{\frac{2 n-1}{2}} \frac{1}{(n-1) !} \frac{\Gamma\left(\frac{\alpha}{2}+\frac{1}{2 \nu}(2 n-1)\right)}{\Gamma\left(\frac{\alpha}{2}+\frac{(1-\nu)}{2 \nu}(2 n-1)\right)}
$$

and

$$
\Omega_{2 r-1,2 s-1}(p, \alpha)=-\Theta\left(\frac{i(2 r-1)}{2 \nu}, \frac{i(2 s-1)}{2 \nu} \mid p, \alpha\right)\left(\frac{r+s-1}{\nu}\right)\left(\frac{\sqrt{2} p \nu}{R}\right)^{2 r+2 s-2} .
$$

The relations (4.8)-(4.11) look the same as (11.5)-(11.7) of [4]. However there is an important difference. It was pointed out in [4] that the expansion coefficients $\Theta_{n}\left(\frac{i(2 r-1)}{2 \nu}, \frac{i(2 s-1)}{2 \nu} \mid p, \alpha\right)$ satisfy the so-called vanishing property i.e. for given $r$ and $s$ they vanish starting from $2 n=r+s$. It is equivalent to the fact that the function $\Theta$ is proportional to a polynomial with respect to $p$. This is true only for the case of ground state $k=0$ and also for the case $k=1$ with $I^{(+, 1)}=I^{(-, 1)}=1$. For both cases all the coefficients $\Theta_{n}$ with odd $n$ vanish. We will see that in both cases the space of the CFT-descendants is one-dimensional.

\section{Relation to the CFT}

Here we would like to study generalization of the relation (2.1) between the lattice six vertex model and the CFT to the case of excited states. As discussed in Section 2, for the case of the ground state we inserted at $\pm \infty$-points of the cylinder the two primary fields $\phi_{ \pm}$with dimensions $\Delta_{ \pm}$. We also identified $\Delta_{+}=\Delta_{\kappa+1}, \Delta_{-}=\Delta_{-\kappa^{\prime}+1}$ and $\Delta_{+}=\Delta_{-}$for the case $\kappa=\kappa^{\prime}$. The corresponding states were denoted $\left|\Delta_{+}\right\rangle$and $\left\langle\Delta_{-}\right|$respectively. For the six vertex model we introduced in Section 3 the states $\left|\kappa ; I^{(+, k)}, I^{(-, k)}\right\rangle$ marked by the two ordered sets $I^{(+, k)}$ and $I^{(-, k)}$ of $k$ odd, positive, non-coinciding numbers. In case of the spin $s$ sector we denote such a state $\left|\kappa, s ; I^{(+, k)}, I^{(-, k)}\right\rangle$. In the scaling limit we can identify these states with descendants of the primary fields $\phi_{ \pm}$at level $N=\frac{1}{2} \sum_{r=1}^{k}\left(I_{r}^{(+, k)}+I_{r}^{(-, k)}\right)$

$$
\begin{aligned}
& \left|\kappa ; I^{(+, k)}, I^{(-, k)}\right\rangle \rightarrow_{\text {scal }}\left|\Delta_{+} ; I^{(+, k)}, I^{(-, k)}\right\rangle=\sum_{\substack{n_{1} \geq \cdots \geq n_{m} \geq 1 \\
n_{1}+\cdots+n_{m}=N}} A_{n_{1}, \ldots, n_{m}}^{\left(I^{(+, k)}, I^{(-, k)}\right)} L_{-n_{1}} \cdots L_{-n_{m}}\left|\Delta_{+}\right\rangle \\
& \quad\left\langle\kappa+\alpha-s, s ; I^{(+, k)}, I^{(-, k)}\right| \rightarrow_{\text {scal }}\left\langle\Delta_{-} ; I^{(+, k)}, I^{(-, k)}\right| \\
& \quad=\sum_{\substack{n_{1} \geq \cdots \geq n_{m} \geq 1 \\
n_{1}+\cdots+n_{m}=N}} \bar{A}_{n_{1}, \ldots, n_{m}}^{\left(I^{(+, k)}, I^{(-, k)}\right)}\left\langle\Delta_{-}\right| L_{n_{m}} \cdots L_{n_{1}} .
\end{aligned}
$$


We normalize

$$
\left\langle\Delta_{-} ; I^{(+, k)}, I^{(-, k)}\left|\phi_{\alpha}(0)\right| \Delta_{+} ; I^{(+, k)}, I^{(-, k)}\right\rangle=1
$$

but we do not demand orthogonality of the states with different sets. In other words, the "scalar product" $\left\langle\Delta_{-} ; I^{(+, k)}, I^{(-, k)}\left|\phi_{\alpha}(0)\right| \Delta_{+} ; I^{\prime(+, k)}, I^{\prime(-, k)}\right\rangle$ is not necessarily zero. So, instead of (2.1) we take

$$
\begin{aligned}
& \sum_{\substack{n_{1} \geq \cdots \geq n_{m} \geq 1 \\
n_{1}+\cdots+n_{m}=N}} \sum_{\substack{n_{1}^{\prime} \geq \cdots \geq n^{\prime}, \geq 1 \\
n_{1}^{\prime}+\cdots+n_{m^{\prime}}^{\prime}=N}} A_{n_{1}, \ldots, n_{m}}^{\left(I^{(+, k)}, I^{(-, k)}\right)} \bar{A}_{n_{1}^{\prime}, \ldots, n_{m^{\prime}}^{\prime}}^{\left(I^{(+, k)}, I^{(-, k)}\right)} \\
& \quad \times\left\langle\Delta_{-}\left|L_{n_{m^{\prime}}^{\prime}} \cdots L_{n_{1}^{\prime}} P_{\alpha}\left(\left\{\mathbf{l}_{-j}\right\}\right) \phi_{\alpha}(0) L_{-n_{1}} \cdots L_{-n_{m}}\right| \Delta_{+}\right\rangle \\
& =\lim _{\substack{\mathbf{n} \rightarrow \infty \\
a \rightarrow 0, \mathbf{n} a=2 \pi R}} \frac{\left\langle\kappa+\alpha-s, s ; I^{(+, k)}, I^{(-, k)}\left|T_{\mathrm{S}, \mathbf{M}} q^{2 \kappa S^{\prime}} \mathbf{b}_{\infty, s-1}^{*} \cdots \mathbf{b}_{\infty, 0}^{*}\left(q^{2 \alpha S(0)} \mathcal{O}\right)\right| \kappa ; I^{(+, k)}, I^{(-, k)}\right\rangle}{\left\langle\kappa+\alpha-s, s ; I^{(+, k)}, I^{(-, k)}\left|T_{\mathrm{S}, \mathbf{M}} q^{2 \kappa S} \mathbf{b}_{\infty, s-1}^{*} \cdots \mathbf{b}_{\infty, 0}^{*}\left(q^{2 \alpha S(0)}\right)\right| \kappa ; I^{(+, k)}, I^{(-, k)}\right\rangle} .
\end{aligned}
$$

In this relation the polynomial $P_{\alpha}\left(\left\{\mathbf{l}_{-j}\right\}\right)$ does not depend on $\kappa$ and the choice of the excitation i.e. it is independent of $k$ and of the both sets $I^{( \pm, k)}$. As was pointed out above, the coefficients of this polynomial are rational functions of the conformal charge $c$ and the conformal dimension $\Delta_{\alpha}$ of the primary field $\phi_{\alpha}$ only. If the operator $\mathcal{O}=\boldsymbol{\beta}_{2 i_{1}-1}^{\mathrm{CFT} *} \cdots \boldsymbol{\beta}_{2 i_{n}-1}^{\mathrm{CFT} *} \boldsymbol{\gamma}_{2 j_{n}-1}^{\mathrm{CFT} *} \cdots \boldsymbol{\gamma}_{2 j_{1}-1}^{\mathrm{CFT} *}$ then the level of descendants participating in $P_{\alpha}\left(\left\{\mathbf{l}_{-j}\right\}\right)$ is $M=2 \sum_{l=1}^{n}\left(i_{l}+j_{l}-1\right)$. On the other hand, the coefficients $A_{n_{1}, \ldots, n_{m}}, \bar{A}_{n_{1}, \ldots, n_{m}}$ are independent of the choice of the operator $\mathcal{O}$. So, our strategy is to take for a given level $M$ all linear independent operators $\mathcal{O}$ represented in terms of the fermionic basis modulo integrals of motion and as many different excitations as necessary in order to fix the corresponding polynomials $P_{\alpha}\left(\left\{\mathbf{l}_{-j}\right\}\right)$ and the coefficients ${ }^{9} A_{n_{1}, \ldots, n_{m}}, \bar{A}_{n_{1}, \ldots, n_{m}}$. All other relations for this level partially determine further coefficients $A, \bar{A}$ and the rest of the equations fulfills automatically. In [4] we were able to fix the polynomials $P_{\alpha}\left(\left\{\mathbf{l}_{-j}\right\}\right)$ up to $M=6$ using only the ground state data when $k=0$. It means that for $M \leq 6$ the relation (5.3) fulfills automatically for any excitation i.e. for any $k$ and any two sets $I^{( \pm, k)}$. We checked this for the case $k=1$ with $I_{1}^{(+, 1)}=I_{1}^{(-, 1)}=1$ and $I_{1}^{(+, 1)}=1, I_{1}^{(-, 1)}=3, I_{1}^{(+, 1)}=3, I_{1}^{(-, 1)}=1$.

Starting with $M=8$ the situation changes. We do not have enough equations in order to fix the polynomials $P_{\alpha}\left(\left\{\mathbf{l}_{-j}\right\}\right)$ if we restrict ourselves with the case of the ground state. We need additional equations involving excitations. In the next section we will consider in detail the case $M=8$.

Before we go further let us make one remark. It is interesting to note that the above fermionic basis operators are marked in exactly the same way as the particle-hole excitations, namely, by two ordered sets of $n$ odd, positive integers $I^{(+, n)}=\left\{2 i_{1}-1<\cdots<2 i_{n}-1\right\}$ and $I^{(-, n)}=$ $\left\{2 j_{1}-1<\cdots<2 j_{n}-1\right\}$. So, we can denote such an operator

$$
\mathcal{O}_{I^{(+, n)}, I^{(-, n)}}=\boldsymbol{\beta}_{2 i_{1}-1}^{\mathrm{CFT} *} \cdots \boldsymbol{\beta}_{2 i_{n}-1}^{\mathrm{CFT} *} \boldsymbol{\gamma}_{2 j_{n}-1}^{\mathrm{CFT} *} \cdots \boldsymbol{\gamma}_{2 j_{1}-1}^{\mathrm{CFT} *} .
$$

In case we take this operator in the right hand side of (5.3) we will use the following shorthand notation

$$
\text { r.h.s. of }(5.3):=\left\langle I^{(+, k)}, I^{(-, k)}\left|\mathcal{O}_{I^{(+, n)}, I^{(-, n)}}\right| I^{(+, k)}, I^{(-, k)}\right\rangle \text {. }
$$

Now let us describe in general how we compute both sides of the relation (5.3). We start with the right hand side of (5.3) determined by the lattice data. We pointed out the fact that

\footnotetext{
${ }^{9}$ In fact, not all coefficients $A, \bar{A}$ can be fixed but rather some of their products. We checked for one particular case that one can fix them completely taking $\bar{A}_{n_{1}, \ldots, n_{m}}=A_{n_{1}, \ldots, n_{m}}$ but a'priori it is not quite clear to us why it should be so.
} 
the theorem by Jimbo, Miwa, Smirnov works for the case of excited states also with the same fermionic operators. Only two transcendental functions $\rho$ and $\omega$ are sensible to the changes that happen in the Matsubara direction. It means that we still can apply the Wick theorem and after taking the scaling limit come to the same determinant formula (2.17) but with a new functional determined by the right hand side of (5.3) instead of $Z_{R}^{\kappa, \kappa^{\prime}}$. With our choice of parameters $\alpha, s$, $\kappa, \kappa^{\prime}$ the function $\rho^{\mathrm{sc}}$ is still 1 and the function $\omega^{\mathrm{sc}}$ is now determined through the asymptotic expansion (4.9). So, we come to

$$
\left\langle I^{(+, k)}, I^{(-, k)}\left|\mathcal{O}_{I^{(+, n)}, I^{(-, n)}}\right| I^{(+, k)}, I^{(-, k)}\right\rangle=\operatorname{det}\left(\frac{\Omega_{2 i_{r}-1,2 j_{r^{\prime}}-1}(p, \alpha)}{i_{r}+j_{r^{\prime}}-1}\right)_{r, r^{\prime}=1, \ldots, n},
$$

where the function $\Omega$ is defined by (4.11).

Now let us proceed to the left hand side of (5.3) which involves the CFT data. For simplicity let us put the radius of the cylinder $R=1$. We need to calculate

$$
\left\langle\Delta_{-}\left|L_{n_{m^{\prime}}^{\prime}} \cdots L_{n_{1}^{\prime}} P_{\alpha}\left(\left\{\mathbf{l}_{-j}\right\}\right) \phi_{\alpha}(0) L_{-n_{1}} \cdots L_{-n_{m}}\right| \Delta_{+}\right\rangle
$$

with $n_{1} \geq \cdots \geq n_{m} \geq 1, n_{1}^{\prime} \geq \cdots \geq n_{m^{\prime}}^{\prime} \geq 1$ and

$$
N=\sum_{j=1}^{m} n_{j}=\sum_{j=1}^{m^{\prime}} n_{j}^{\prime}
$$

or picking out some monomial with respect to the local Virasoro generators $\mathbf{l}_{-j}$, we need the following value

$$
\begin{aligned}
& \left(n_{1}^{\prime}, \ldots, n_{m^{\prime}}^{\prime} ; a_{1}, \ldots, a_{d} ; n_{1}, \ldots, n_{m}\right) \\
& \quad:=\left\langle\Delta_{-}\left|L_{n_{m^{\prime}}^{\prime}} \cdots L_{n_{1}^{\prime}} \mathbf{l}_{-2 a_{d}} \cdots \mathbf{l}_{-2 a_{1}} \phi_{\alpha}(0) L_{-n_{1}} \cdots L_{-n_{m}}\right| \Delta_{+}\right\rangle
\end{aligned}
$$

with $d$ positive integers $1 \leq a_{1} \leq \cdots \leq a_{d}$.

In order to compute it we follow the scheme described in Section 6 of [4]. First we define the function

$$
W\left(z_{1}, \ldots, z_{K} ; w\right):=\left\langle\Delta_{-}\left|T\left(z_{K}\right) \cdots T\left(z_{1}\right) \phi_{\alpha}(w)\right| \Delta_{+}\right\rangle, \quad K=d+m+m^{\prime},
$$

where $T(z)$ is the energy-momentum tensor as a function of the point $z$ on the cylinder with the OPEs

$$
\begin{aligned}
& T(z) T(w)=-\frac{c}{12} \chi^{\prime \prime \prime}(z-w)-2 T(w) \chi^{\prime}(z-w)+T^{\prime}(w) \chi(z-w)+O(1), \\
& T(z) \phi_{\alpha}(w)=-\Delta_{\alpha} \phi_{\alpha}(w) \chi^{\prime}(z-w)+\phi_{\alpha}^{\prime}(w) \chi(z-w)+O(1)
\end{aligned}
$$

and

$$
\chi(z)=\frac{1}{2} \operatorname{coth}\left(\frac{z}{2}\right)=\sum_{n=0}^{\infty} \frac{B_{2 n}}{(2 n) !} z^{2 n-1},
$$

where $B_{2 n}$ are Bernulli numbers. We also need the expansion:

$$
\chi(z)= \pm \frac{1}{2} \pm \sum_{j=1}^{\infty} e^{\mp j z}, \quad \Re(z) \rightarrow \pm \infty .
$$

As was discussed above we can use two different expansions for the energy-momentum tensor as well 
- the "local" expansion in vicinity of $z=0$

$$
T(z)=\sum_{n=-\infty}^{\infty} \mathbf{l}_{n} z^{-n-2},
$$

- the "global" expansion when $\Re(z) \rightarrow \pm \infty$

$$
T(z)=\sum_{n=-\infty}^{\infty} L_{n} e^{n z}-\frac{c}{24} .
$$

The action of the local Virasoro generators $\mathbf{l}_{n}$ on a local field $O(w)$ is defined through the contour integral

$$
\left(\mathbf{l}_{n} O\right)(w)=\int_{C_{w}} \frac{d z}{2 \pi i}(z-w)^{n+1} T(z) O(w),
$$

where $C_{w}$ encircles the point $w$ anticlockwise.

The conformal Ward-Takahashi identity allows to determine the function $W\left(z_{1}, \ldots, z_{K}\right)$ recursively:

$$
\begin{aligned}
& W\left(z_{1}, \ldots, z_{K} ; w\right)=-\frac{c}{12} \sum_{j=2}^{K} \chi^{\prime \prime \prime}\left(z_{1}-z_{j}\right) W\left(z_{2}, \ldots \stackrel{{ }^{j}}{\wedge} \ldots, z_{K} ; w\right) \\
& \quad+\left\{\sum_{j=2}^{K}\left(-2 \chi^{\prime}\left(z_{1}-z_{j}\right)+\left(\chi\left(z_{1}-z_{j}\right)-\chi\left(z_{1}-w\right)\right) \frac{\partial}{\partial z_{j}}\right)-\Delta_{\alpha} \chi^{\prime}\left(z_{1}-w\right)\right. \\
& \left.\quad+\left(\Delta_{+}-\Delta_{-}\right) \chi\left(z_{1}-w\right)+\frac{1}{2}\left(\Delta_{+}+\Delta_{-}\right)-\frac{c}{24}\right\} W\left(z_{2}, \ldots, z_{K} ; w\right) .
\end{aligned}
$$

Of course, the term containing the difference $\Delta_{+}-\Delta_{-}$drops for the case $\Delta_{+}=\Delta_{-}$which is only interesting for us here.

In order to calculate the above object $\left(n_{1}^{\prime}, \ldots, n_{m^{\prime}}^{\prime} ; a_{1}, \ldots, a_{d} ; n_{1}, \ldots, n_{m}\right)$ given by $(5.6)$, we proceed in several steps:

step 1: take the recursion (5.10) and expand $\chi\left(z_{1}-\cdots\right), \chi^{\prime}\left(z_{1}-\cdots\right), \chi^{\prime \prime}\left(z_{1}-\cdots\right), \chi^{\prime \prime \prime}\left(z_{1}-\cdots\right)$ using the expansion (5.8) for $\Re\left(z_{1}\right) \rightarrow-\infty$ and then having in mind the expansion (5.9) take there the coefficient at $e^{n_{1}^{\prime} z_{1}}$,

step 2: repeat this procedure consequently for the variables $z_{2}, z_{3}, \ldots, z_{m^{\prime}}$ taking every time the coefficients at $e^{n_{2}^{\prime} z_{2}}, e^{n_{3}^{\prime} z_{3}}, \ldots, e^{n_{m^{\prime}}^{\prime} z_{m^{\prime}}}$,

step 3: similarly we proceed with the next $m$ variables $z_{m^{\prime}+1}, \ldots, z_{m^{\prime}+m}$ taking the expansion (5.8) for $\Re\left(z_{m^{\prime}+1}\right) \rightarrow \infty, \ldots, \Re\left(z_{m^{\prime}+m}\right) \rightarrow \infty$, further using the recursion (5.10) and picking up coefficients at $e^{-n_{1} z_{m^{\prime}+1}}, \ldots, e^{-n_{m} z_{m^{\prime}+m}}$,

step 4: now one can easily compute the limit $w \rightarrow 0$ and then apply (5.10) with respect to the variable $z_{m^{\prime}+m+1}$, take the local expansion (5.7) of $\chi\left(z_{m^{\prime}+m+1}\right), \chi^{\prime}\left(z_{m^{\prime}+m+1}\right)$, $\chi^{\prime \prime}\left(z_{m^{\prime}+m+1}\right), \ldots$ and calculate the contour integral $\int_{C_{0}} d z_{m^{\prime}+m+1} z_{m^{\prime}+m+1}^{-2 a_{1}+1} \ldots$ with the expression obtained in this way,

step 5: repeat the step 4 with respect to the residual variables $z_{m^{\prime}+m+2}, \ldots, z_{K}$ every time calculating the contour integrals $\int_{C_{0}} d z_{m^{\prime}+m+2} z_{m^{\prime}+m+2}^{-2 a_{2}+1} \cdots$ etc. up to the last integral $\int_{C_{0}} d z_{K} z_{K}^{-2 a_{d}+1} \cdots$. 
In this way we can obtain, for example, for the case $d=0, N=2$

$$
\begin{aligned}
& (2 ; \varnothing ; 2)=\frac{c}{2}+4\left(\Delta^{2}-\Delta+\Delta_{+}\right), \\
& (1,1 ; \varnothing ; 2)=(2 ; \varnothing ; 1,1)=2\left(\Delta^{3}-\Delta+3 \Delta_{+}\right), \\
& (1,1 ; \varnothing ; 1,1)=\Delta(\Delta-1)\left(\Delta^{2}-\Delta+2\right)+4 \Delta_{+}\left(2 \Delta^{2}-2 \Delta+1\right)+8 \Delta_{+}^{2},
\end{aligned}
$$

where again we used the shorthand notation $\Delta \equiv \Delta_{\alpha}$.

For $d=1, a_{1}=1, N=2$ we get

$$
\begin{aligned}
(2 ; 1 ; 2)= & -\frac{1}{3} \Delta\left(\Delta^{2}-61 \Delta+42\right)+c\left(-\frac{1}{6} \Delta^{2}+\frac{17}{8} \Delta+1\right)-\frac{c^{2}}{48} \\
+ & \Delta_{+}\left(4 \Delta^{2}+\frac{35}{3} \Delta+\frac{c}{3}+8\right)+4 \Delta_{+}^{2}, \\
(1,1 ; 1 ; 2)= & (2 ; 1 ; 1,1)=-\frac{1}{12} \Delta(\Delta+1)\left(2 \Delta^{2}+c \Delta-86 \Delta+72-7 c\right) \\
& +\Delta_{+}\left((2 \Delta+3)\left(\Delta^{2}+\frac{9}{2} \Delta+4\right)-\frac{c}{4}\right)+6 \Delta_{+}^{2}, \\
(1,1 ; 1 ; 1,1)= & -\frac{1}{24} \Delta(\Delta-1)\left(2 \Delta^{3}+(c-50) \Delta^{2}-(c+44) \Delta+2 c-96\right) \\
& +\frac{\Delta_{+}}{6}\left(6 \Delta^{4}+32 \Delta^{3}-2(c-71) \Delta^{2}+2(c+29) \Delta+48-c\right) \\
& +\frac{\Delta_{+}^{2}}{3}\left(24 \Delta^{2}+70 \Delta+60-c\right)+8 \Delta_{+}^{3} .
\end{aligned}
$$

And for $d=1, a_{1}=2, N=2$ :

$$
\begin{aligned}
&(2 ; 2 ; 2)=\Delta\left(\frac{\Delta^{2}}{60}+\frac{359}{60} \Delta+\frac{1921 c}{480}-3\right)+\frac{1921 \Delta \Delta_{+}}{60} \\
&(1,1 ; 2 ; 2)=\Delta(\Delta+1)\left(\frac{\Delta^{2}}{120}+\frac{179}{120} \Delta+c-1\right)+\Delta\left(12 \Delta+\frac{1441}{40}\right) \Delta_{+}, \\
&(1,1 ; 2 ; 1,1)=\Delta^{2}(\Delta-1)\left(\frac{\Delta(\Delta-1)}{240}+\frac{241}{120}\right)+\Delta\left(\frac{121}{30} \Delta^{2}+\frac{599}{30} \Delta+\frac{1921}{60}\right) \Delta_{+} \\
& \quad+\frac{481 \Delta \Delta_{+}^{2}}{30} .
\end{aligned}
$$

\section{The level $M=8$}

Here we show how the procedure generally described in the previous section works for the case of level $M=8$. As was pointed out in Introduction, there are 5 monomials of the local Virasoro generators which generate linear independent descendant states modulo integrals of motion. Let us arrange them as a vector

$$
V_{\mathbf{l}}=\left(\begin{array}{c}
\mathbf{l}_{-2}^{4} \\
\mathbf{l}_{-4} \mathbf{l}_{-2}^{2} \\
\mathbf{l}_{-4}^{2} \\
\mathbf{l}_{-6} \mathbf{l}_{-2} \\
\mathbf{l}_{-8}
\end{array}\right)
$$


As for the fermionic operators (5.4), we also have 5 possibilities for $M=8$ which we also take as a vector

$$
V_{\text {fermi }}=\left(\begin{array}{c}
\phi_{1,7}^{\text {even }} \\
\phi_{1,7}^{\text {odd }} \\
\phi_{3,5}^{\text {even }} \\
\phi_{3,5}^{\text {odd }} \\
\boldsymbol{\beta}_{1}^{\mathrm{CFT} *} \boldsymbol{\beta}_{3}^{\mathrm{CFT} *} \gamma_{3}^{\mathrm{CFT} *} \boldsymbol{\gamma}_{1}^{\mathrm{CFT} *}
\end{array}\right) \text {, }
$$

where we used the even and odd combinations (2.18). We would like to determine the 5 by 5 transformation matrix $U$

$$
V_{\text {fermi }} \cong U V_{l},
$$

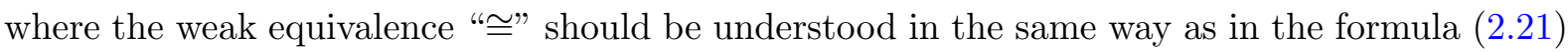
and all the matrix elements of $U$ depend only on the conformal dimension $\Delta \equiv \Delta_{\alpha}$ and central charge $c$. In [4] we were not able to uniquely fix this matrix from the consideration of the ground state case $N=0$. If we substitute the both sides of (6.1) into (2.1) where the matrix elements of $U$ in every row correspond to the coefficients in the polynomial $P_{\alpha}\left(\left\{\mathbf{1}_{-k}\right\}\right)$ and use (5.5) for $k=0$ then we get

$$
\frac{\left\langle\Delta_{-}\left|\left(U V_{1}\right) \phi_{\alpha}(0)\right| \Delta_{+}\right\rangle}{\left\langle\Delta_{-}\left|\phi_{\alpha}(0)\right| \Delta_{+}\right\rangle}=V^{(0)}
$$

where

$$
V^{(0)}:=\left(\begin{array}{c}
\left(\Omega_{1,7}^{(0)}+\Omega_{7,1}^{(0)}\right) / 2 \\
\left(\Omega_{7,1}^{(0)}-\Omega_{1,7}^{(0)}\right) /\left(2 d_{\alpha}\right) \\
\left(\Omega_{3,5}^{(0)}+\Omega_{5,3}^{(0)}\right) / 2 \\
\left(\Omega_{5,3}^{(0)}-\Omega_{3,5}^{(0)}\right) /\left(2 d_{\alpha}\right) \\
\Omega_{1,1}^{(0)} \Omega_{3,3}^{(0)} / 3-\Omega_{1,3}^{(0)} \Omega_{3,1}^{(0)} / 4
\end{array}\right)
$$

and $\Omega_{j, j^{\prime}}^{(0)}$ denote the functions $\Omega_{j, j^{\prime}}(p, \alpha)$ given by (4.11) which are calculated for the ground state case $N=0, k=0$ as was explained in Section 11 of [4]. From the vanishing property follows that all five equations in (6.2) are polynomials with respect to $p^{2}$ of degree 4 . In all 5 cases one of the equations is fulfilled automatically. It means that every equation leaves an one-parametric freedom. In other words, in every row of $U$ one matrix element is left undetermined.

Therefore in order to fix the matrix $U$ completely we need to involve excitations. Here we restrict ourselves with the case $k=1$ and $N \leq 2$ only. Let us remind the reader that the descendant level for the excitations $N=\frac{1}{2} \sum_{r=1}^{k}\left(I_{r}^{(+, k)}+I_{r}^{(-, k)}\right)$. So, for the case $N=1$ we have only one possibility $I^{(+, 1)}=I^{(-, 1)}=\{1\}$. As in the ground state case $N=0$, the descendant space is one-dimensional because there is only one vector $L_{-1}\left|\Delta_{+}\right\rangle$here. When we wrote the paper [4] we thought that considering this excitation would help us to fix the above mentioned freedom. As appeared it is not the case because it does not produce any additional constraints on the transformation matrix $U$. We leave the checking of this fact as an exercise for the reader.

So, we have to consider the case $N=2$. There are two possibilities $I^{(+, 1)}=\{1\}, I^{(-, 1)}=\{3\}$ and $I^{(+, 1)}=\{3\}, I^{(-, 1)}=\{1\}$ that correspond to the two-dimensional descendant space spanned by two vectors $L_{-2}\left|\Delta_{+}\right\rangle$and $L_{-1}^{2}\left|\Delta_{+}\right\rangle$. Now let us consider the states (5.1)

$$
\left|\Delta_{+} ;\{1\},\{3\}\right\rangle=A L_{-2}\left|\Delta_{+}\right\rangle+B L_{-1}^{2}\left|\Delta_{+}\right\rangle,
$$




$$
\begin{aligned}
& \left|\Delta_{+} ;\{3\},\{1\}\right\rangle=C L_{-2}\left|\Delta_{+}\right\rangle+D L_{-1}^{2}\left|\Delta_{+}\right\rangle, \\
& \left\langle\Delta_{-} ;\{1\},\{3\}\right|=\bar{A}\left\langle\Delta_{-}\right| L_{2}+\bar{B}\left\langle\Delta_{-}\right| L_{1}^{2}, \\
& \left\langle\Delta_{-} ;\{3\},\{1\}\right|=\bar{C}\left\langle\Delta_{-}\right| L_{2}+\bar{D}\left\langle\Delta_{-}\right| L_{1}^{2},
\end{aligned}
$$

where we use simpler notation for the coefficients $A_{2}^{(\{1\},\{3\})} \equiv A, A_{1,1}^{(\{1\},\{3\})} \equiv B, A_{2}^{(\{3\},\{1\})} \equiv C$, $A_{1,1}^{(\{3\},\{1\})} \equiv D$ and similar for $\bar{A}, \bar{B}, \bar{C}, \bar{D}$. As appeared we can take them $\bar{A}=A, \bar{B}=B$, $\bar{C}=C, \bar{D}=D$.

Now we should satisfy the normalization condition (5.2)

$$
\left\langle\Delta_{-} ;\{1\},\{3\}\left|\phi_{\alpha}(0)\right| \Delta_{+} ;\{1\},\{3\}\right\rangle=\left\langle\Delta_{-} ;\{3\},\{1\}\left|\phi_{\alpha}(0)\right| \Delta_{+} ;\{3\},\{1\}\right\rangle=1 .
$$

Substituting the above formulae (6.4) here, using the notation $(5.6)$ and the fact that $(1,1 ; \varnothing ; 2)=$ $(2 ; \varnothing ; 1,1)$, we get

$$
\begin{aligned}
& A^{2}(2 ; \varnothing ; 2)+B^{2}(1,1 ; \varnothing ; 1,1)+2 A B(1,1 ; \varnothing ; 2) \\
& \quad=C^{2}(2 ; \varnothing ; 2)+D^{2}(1,1 ; \varnothing ; 1,1)+2 C D(1,1 ; \varnothing ; 2)=1,
\end{aligned}
$$

where $(2 ; \varnothing ; 2),(1,1 ; \varnothing ; 1,1),(1,1 ; \varnothing ; 2)$ are given by $(5.11)$. Now we can use the first row of (2.21), the formulae (5.5) and (6.5) in order to obtain

$$
\left(\begin{array}{ccc}
(2 ; \varnothing ; 2) & (1,1 ; \varnothing ; 1,1) & (1,1 ; \varnothing ; 2) \\
(2 ; 1 ; 2) & (1,1 ; 1 ; 1,1) & (1,1 ; 1 ; 2) \\
(2 ; 2 ; 2) & (1,1 ; 2 ; 1,1) & (1,1 ; 2 ; 2)
\end{array}\right)\left(\begin{array}{c}
A^{2} \\
B^{2} \\
2 A B
\end{array}\right)=\left(\begin{array}{c}
1 \\
\Omega_{1,1}^{(1,3)} \\
\frac{3}{4}\left(\Omega_{3,1}^{(1,3)}-\Omega_{1,3}^{(1,3)}\right) / d_{\alpha}
\end{array}\right)
$$

Here we denoted $\Omega_{j, j^{\prime}}^{(1,3)}$ the function $\Omega_{j, j^{\prime}}(p, \alpha)$ given by the formula (4.11) where

$$
\Theta\left(i j /(2 \nu), i j^{\prime} /(2 \nu) \mid p, \alpha\right)
$$

is determined by the equation (4.7) with $k=1$ and $I^{(+, 1)}=\{1\}, I^{(-, 1)}=\{3\}$ while $d_{\alpha}$ is taken from (2.19). The matrix elements of the second and the third row in the left hand side of (6.6) are given by (5.12) and (5.13) respectively. The matrix equation (6.6) can be solved with respect to $A$ and $B$ by inverting the 3 by 3 matrix. These equations are overdetermined. The first two of them give $A^{2}, B^{2}$ and we can check that the last equation which gives $2 A B$ is fulfilled automatically.

In a similar way one can get the coefficients $C$ and $D$ from the equation which can be obtained from (6.6) by changing $A \rightarrow C, B \rightarrow D$ and $\Omega_{j, j^{\prime}}^{(1,3)} \rightarrow \Omega_{j, j^{\prime}}^{(3,1)}$ where $\Omega_{j, j^{\prime}}^{(3,1)}$ is defined through the equations (4.11) and (4.7) with $I^{(+, 1)}=\{3\}, I^{(-, 1)}=\{1\}$.

The result for few leading orders with respect to $p$ looks as follows

$$
\begin{aligned}
& A=\frac{1}{4}(p \nu)^{-1}+\frac{c-22}{192}(p \nu)^{-2}-\left(\frac{\Delta(\Delta-1)}{16}+\frac{c^{2}+148 c-860}{18432}\right)(p \nu)^{-3}+O\left(p^{-4}\right), \\
& B=\frac{1}{8}(p \nu)^{-2}-\frac{c+14}{384}(p \nu)^{-3}+O\left(p^{-4}\right) .
\end{aligned}
$$

The coefficients $C$ and $D$ can be got from $A$ and $B$ respectively through the substitution of $p$ by $-p$.

As in the $N=0$ case described above, one can substitute (6.1) into (5.3) and use (5.5) in order to get

$$
A^{2}\left\langle\Delta_{-}\left|L_{2}\left(U V_{\mathbf{l}}\right) \phi_{\alpha}(0) L_{-2}\right| \Delta_{+}\right\rangle+B^{2}\left\langle\Delta_{-}\left|L_{1}^{2}\left(U V_{\mathbf{l}}\right) \phi_{\alpha}(0) L_{-1}^{2}\right| \Delta_{+}\right\rangle
$$




$$
+2 A B\left\langle\Delta_{-}\left|L_{1}^{2}\left(U V_{1}\right) \phi_{\alpha}(0) L_{-2}\right| \Delta_{+}\right\rangle=V^{(1,3)}
$$

where $V^{(1,3)}$ can be obtained from $V^{(0)}$ given by (6.3) via the substitution $\Omega_{j, j^{\prime}}^{(0)} \rightarrow \Omega_{j, j^{\prime}}^{(1,3)}$

$$
V^{(1,3)}:=\left(\begin{array}{c}
\left(\Omega_{1,7}^{(1,3)}+\Omega_{7,1}^{(1,3)}\right) / 2 \\
\left(\Omega_{7,1}^{(1,3)}-\Omega_{1,7}^{(1,3)}\right) /\left(2 d_{\alpha}\right) \\
\left(\Omega_{3,5}^{(1,3)}+\Omega_{5,3}^{(1,3)}\right) / 2 \\
\left(\Omega_{5,3}^{(1,3)}-\Omega_{3,5}^{(1,3)}\right) /\left(2 d_{\alpha}\right) \\
\Omega_{1,1}^{(1,3)} \Omega_{3,3}^{(1,3)} / 3-\Omega_{1,3}^{(1,3)} \Omega_{3,1}^{(1,3)} / 4
\end{array}\right) .
$$

Both sides of the equation (6.8) can be represented as a power series with respect to $p^{-1}$ starting with $p^{0}$. Then one can equate coefficients standing at powers $p^{-j}$ and get as many equations as necessary. It turned out that all the equations obtained by taking coefficients at $p^{0}$ up to $p^{-8}$ fulfill automatically. The unknown matrix elements of the transformation matrix $U$ are fixed only in the order $p^{-9}$. All equations which stem from further orders with respect to $p^{-1}$ should be fulfilled automatically. Unfortunately, so far we could not prove it or even check any further equations because of complexity of calculations in the intermediate stage. We hope to do it in future. We checked that if one substitutes $A \rightarrow C, B \rightarrow D$ in the left hand side of the equation (6.8) and $\Omega_{j, j^{\prime}}^{(1,3)} \rightarrow \Omega_{j, j^{\prime}}^{(3,1)}$ in the right hand side of (6.8) then the equation obtained is fulfilled automatically up to $p^{-9}$. Let us explicitly show the final result using even and odd combinations (2.18):

$$
\begin{aligned}
\phi_{1,7}^{\text {even }} \cong & \mathbf{l}_{-2}^{4}+(4(c-4) \Delta+4(c+8)) /(3(\Delta+4)) \mathbf{l}_{-4} \mathbf{l}_{-2}^{2} \\
& -\left(-43 c^{2}+924 c-16340-\left(21 c^{2}+1102 c-18535\right) \Delta\right. \\
& \left.-\left(11 c^{2}-198 c+775\right) \Delta^{2}+40(c-25) \Delta^{3}\right) /(45(\Delta+4)(\Delta+11)) \mathbf{l}_{-4}^{2} \\
& -\left(2\left(-c^{2}-1540 c+17264\right)-2\left(43 c^{2}-298 c+2652\right) \Delta\right. \\
& \left.-12\left(c^{2}-31 c+574\right) \Delta^{2}+32(c-28) \Delta^{3}\right) /(15(\Delta+4)(\Delta+11)) \mathbf{l}_{-6} \mathbf{l}_{-2} \\
& -\left(-45 c^{3}+1637 c^{2}-137176 c+2033360-20\left(2 c^{3}+261 c^{2}-7623 c+67411\right) \Delta\right. \\
& -4\left(10 c^{3}-439 c^{2}+7142 c-5825\right) \Delta^{2} \\
& \left.+96\left(2 c^{2}-81 c+705\right) \Delta^{3}\right) /(105(\Delta+4)(\Delta+11)) \mathbf{l}_{-8}, \\
\phi_{1,7}^{\text {odd }} \cong & (4 \Delta) /(\Delta+4) \mathbf{l}_{-4} \mathbf{l}_{-2}^{2} \\
& +\left(20(c-28)+24(c+47) \Delta+8(2 c-11) \Delta^{2}\right) /(15(\Delta+4)(\Delta+11)) \mathbf{l}_{-4}^{2} \\
& +\left(-40(c-28)+8(13 c-44) \Delta+16(c-8) \Delta^{2}\right) /(5(\Delta+4)(\Delta+11)) \mathbf{l}_{-6} \mathbf{l}_{-2} \\
& -\left(20(c-28)(c+79)-4\left(15 c^{2}+1316 c-20143\right) \Delta\right. \\
& \left.-4\left(15 c^{2}-398 c+4359\right) \Delta^{2}+32(c-33) \Delta^{3}\right) /(35(\Delta+4)(\Delta+11)) \mathbf{l}_{-8}, \\
\phi_{3,5}^{\text {even }} \cong & \mathbf{l}_{-2}^{4}+(8(2 c+13)+12(c-16) \Delta) /(9(\Delta+4)) \mathbf{l}_{-4} \mathbf{l}_{-2}^{2} \\
& +\left(2\left(262 c^{2}-4271 c+82750\right)+3\left(59 c^{2}+2338 c-67785\right) \Delta\right. \\
& \left.+\left(79 c^{2}-1502 c-6965\right) \Delta^{2}+120(c-25) \Delta^{3}\right) /(405(\Delta+4)(\Delta+11)) \mathbf{l}_{-4}^{2} \\
& +\left(4\left(68 c^{2}+7571 c-86380\right)+4\left(188 c^{2}-3379 c+10310\right) \Delta\right. \\
& \left.+24\left(4 c^{2}-127 c+1125\right) \Delta^{2}\right) /(135(\Delta+4)(\Delta+11)) \mathbf{l}_{-6} \mathbf{l}_{-2} \\
& +\left(2\left(420 c^{3}-9013 c^{2}+711929 c-10449400\right)\right. \\
& +4\left(70 c^{3}+9741 c^{2}-370938 c+3325745\right) \Delta \\
& \\
& +(6)
\end{aligned}
$$




$$
\begin{aligned}
& +20\left(14 c^{3}-629 c^{2}+9532 c-48805\right) \Delta^{2} \\
& \left.+96(c-25)(2 c-31) \Delta^{3}\right) /(945(\Delta+4)(\Delta+11)) \mathbf{l}_{-8} \\
\phi_{3,5}^{\text {odd }} \cong & (4 \Delta) /(3(\Delta+4)) \mathbf{l}_{-4} \mathbf{l}_{-2}^{2} \\
& +\left(-4(17 c-236)-24(c-43) \Delta+8(4 c-127) \Delta^{2}\right) /(135(\Delta+4)(\Delta+11)) \mathbf{l}_{-4}^{2} \\
& +\left(8(17 c-236)+8(47 c-536) \Delta+48(c-18) \Delta^{2}\right) /(45(\Delta+4)(\Delta+11)) \mathbf{l}_{-6} \mathbf{l}_{-2} \\
& +\left(28\left(15 c^{2}-571 c+7708\right)+4\left(35 c^{2}+2708 c-66859\right) \Delta\right. \\
& \left.+20\left(7 c^{2}-222 c+767\right) \Delta^{2}+96(c-33) \Delta^{3}\right) /(315(\Delta+4)(\Delta+11)) \mathbf{l}_{-8}
\end{aligned}
$$

and for the fourth-order combination we get

$$
\begin{aligned}
\beta_{1}^{\mathrm{CFT}} * & \beta_{3}^{\mathrm{CFT} *} \gamma_{3}^{\mathrm{CFT} *} \gamma_{1}^{\mathrm{CFT} *} \cong 1 / 12 \cdot \mathbf{l}_{-2}^{4}+(3 c-54+2(c-22) \Delta) /(18(\Delta+4)) \mathbf{l}_{-4} \mathbf{l}_{-2}^{2} \\
& -\left(43 c^{2}-1426 c+11664+2\left(15 c^{2}-302 c-1069\right) \Delta\right. \\
& \left.+2\left(c^{2}+86 c-2667\right) \Delta^{2}+16(c-25) \Delta^{3}\right) /(216(\Delta+4)(\Delta+11)) \mathbf{l}_{-4}^{2} \\
& +\left(215 c^{2}-8714 c+74952+\left(125 c^{2}-3856 c+13208\right) \Delta\right. \\
& \left.+2\left(5 c^{2}-73 c-1816\right) \Delta^{2}+16(c-28) \Delta^{3}\right) /(180(\Delta+4)(\Delta+11)) \mathbf{l}_{-6} \mathbf{l}_{-2} \\
& -\left(-25 c^{3}+1755 c^{2}-39410 c+326592-4\left(30 c^{2}-1393 c+9520\right) \Delta\right. \\
& \left.+8(11 c+289) \Delta^{2}+192 \Delta^{3}\right) /(72(\Delta+4)(\Delta+11)) \mathbf{l}_{-8} .
\end{aligned}
$$

The elements of the above matrix $U$ can be easily got from these data. It is interesting to note that the determinant of $U$ has relatively simple factorized form

$$
\begin{aligned}
\operatorname{det} U= & -32(c-25)(c-28)(c-33)(\Delta-1) \\
& \times \frac{\left(c+2-2(c+11) \Delta+48 \Delta^{2}\right)\left(-c^{2}+18 c+175+16(c-25) \Delta+192 \Delta^{2}\right)}{382725(\Delta+4)(\Delta+11)} .
\end{aligned}
$$

The numerator of (6.12) is proportional to the following product

$$
\begin{aligned}
& (\nu-2)^{2}(\nu-3)(\nu-4)(2 \nu-3)(3 \nu-4)(\alpha \nu-1)(\alpha \nu-2)(\alpha \nu+1-\nu)(\alpha \nu-1-\nu) \\
& \quad \times(\alpha \nu+2-\nu)(\alpha \nu-2+\nu)(\alpha \nu-2-\nu)(\alpha \nu+1-2 \nu)(\alpha \nu+2-2 \nu)(\alpha \nu+2-3 \nu) .
\end{aligned}
$$

It would be interesting to understand the meaning of the degeneration points like $\nu=2, \nu=3$, $\nu=4, \nu=2 / 3, \nu=3 / 4$ and $\alpha=1 / \nu, \alpha=2 / \nu, \alpha=-(1-\nu) / \nu$ etc. where the determinant $\operatorname{det} U=0$.

\section{Conclusion}

In this paper we demonstrated that the method developed in [4] works for excited states as well. As we saw with the example of the descendant level $M=8$, it is not possible to completely determine the transformation matrix between the usual basis constructed through the action of Virasoro generators on the primary field and the fermionic basis constructed in [1,2] without involving excitations. On the other hand the matrix elements of the transformation matrix should not depend on the fact which excitation is taken. This should provide an interesting compatibility condition on the structure of the three-point functions both from the point of view of the CFT and the lattice XXZ model we started with. In this paper we were able to treat only the case of excitations corresponding to the descendant level $N \leq 2$. It would be interesting to check the above mentioned compatibility for the case of other excitations with $N>2$ and for the higher descendants $M>8$ as well. Of course, it is very important to find 
a general proof of the compatibility condition. We think it is also interesting to study singular points of the transformation matrix mentioned in the end of the previous section. This may shed new light on the structure of the Virasoro algebra, Verma modules and singular vectors. One more important generalization of the results obtained here, which is still out of our reach, would be to treat the case of general values $\alpha, \kappa, \kappa^{\prime}$ and also the case of different excitations inserted at $+\infty$ and $-\infty$ on the cylinder. In both cases the function $\rho$ is not 1 and we would have to generalize the whole Wiener-Hopf factorization technique. We hope to return to these questions in future publications.

\section{A The function $\Phi^{(p)}$ and integrals of motion}

Here we show several further orders of the $1 / p$-expansion of the functions used in Section 3 for the case of excitations with $k=1$. For simplicity we use shorter notation $m_{0} \equiv I_{1}^{(+, 1)}$, $m_{1} \equiv I_{1}^{(-, 1)}$. First let us show expressions for several coefficients $\Psi_{j}^{(p)}(l)$ of the expansion (3.26) for the function $\Psi^{(p)}(l, p)$ :

$$
\begin{aligned}
& \Psi_{0}^{(p)}(l)=-\frac{i}{l(l+i /(2 \nu))}, \\
& \Psi_{1}^{(p)}(l)=0, \\
& \Psi_{2}^{(p)}(l)=\frac{i}{24}\left(-1+12\left(m_{0}+m_{1}\right)\right), \\
& \Psi_{3}^{(p)}(l)=-\frac{l-i /(2 \nu)}{8}\left(m_{0}^{2}-m_{1}^{2}\right) \\
& \Psi_{4}^{(p)}(l)=-\frac{l-i /(2 \nu)}{2880 \nu(1-\nu)}\left(i l \nu(1-\nu)\left(7 / 2+60\left(m_{0}^{3}+m_{1}^{3}\right)\right)+5\left(2 \nu^{2}-11(1-\nu)\right)\left(m_{0}^{3}+m_{1}^{3}\right)\right. \\
& \left.+5(1+\nu)(2 \nu-1)\left(m_{0}+m_{1}\right)\left(6\left(m_{0}+m_{1}\right)-1\right)+\nu^{2}+3(1-\nu)\right), \\
& \Psi_{5}^{(p)}(l)=\frac{l-i /(2 \nu)}{147456 \nu^{2}(1-\nu)^{2}}\left(m_{0}^{2}-m_{1}^{2}\right)\left(384 l^{2} \nu^{2}(1-\nu)^{2}\left(m_{0}^{2}+m_{1}^{2}\right)\right. \\
& -16 i l \nu(1-\nu)\left(5\left(2 \nu^{2}+11(1-\nu)\right)\left(m_{0}^{2}+m_{1}^{2}\right)\right. \\
& \left.+2(1+\nu)(2 \nu-1)\left(12\left(m_{0}+m_{1}\right)-1\right)\right) \\
& -\left(20 \nu^{4}-220 \nu^{3}+681 \nu^{2}-922 \nu+461\right)\left(m_{0}^{2}+m_{1}^{2}\right) \\
& \left.\left.-2(1+\nu)(2 \nu-1)\left(2 \nu^{2}+23(1-\nu)\right)\left(12\left(m_{0}+m_{1}\right)-1\right)\right)\right) \text {, } \\
& \Psi_{6}^{(p)}(l)=\frac{l-i /(2 \nu)}{8360755200 \nu^{3}(1-\nu)^{3}}\left\{8640 i l^{3}\left(-31+252\left(m_{0}^{5}+m_{1}^{5}\right)\right)(1-\nu)^{3} \nu^{3}\right. \\
& +1440 l^{2}(1-\nu)^{2} \nu^{2}\left(567\left(2 \nu^{2}+11(1-\nu)\right)\left(m_{0}^{5}+m_{1}^{5}\right)\right. \\
& +21(1+\nu)(2 \nu-1)\left(m_{0}+m_{1}\right)\left(7-10\left(m_{0}^{2}+m_{1}^{2}-m_{0} m_{1}\right)+120\left(m_{0}^{3}+m_{1}^{3}\right)\right) \\
& \left.-164 \nu^{2}-755(1-\nu)\right)-90 i l \nu(1-\nu)\left(2 1 \left(6085-12170 \nu+8769 \nu^{2}-2684 \nu^{3}\right.\right. \\
& \left.+244 \nu^{4}\right)\left(m_{0}^{5}+m_{1}^{5}\right)+(1+\nu)(2 \nu-1)\left(420\left(34 \nu^{2}+295(1-\nu)\right)\left(m_{0}^{4}+m_{1}^{4}\right)\right. \\
& +840\left(14 \nu^{2}+113(1-\nu)\right)\left(m_{0}^{3} m_{1}+m_{0} m_{1}^{3}\right) \\
& -2520\left(2 \nu^{2}+23(1-\nu)\right) m_{0}^{2} m_{1}^{2}+70\left(274 \nu^{2}-257(1-\nu)\right)\left(m_{0}^{3}+m_{1}^{3}\right) \\
& +2520(1+\nu)(2 \nu-1)\left(m_{0}+m_{1}\right)\left(-m_{0}-m_{1}+12 m_{0} m_{1}\right) \\
& \left.\left.+7\left(158 \nu^{2}+761(1-\nu)\right)\left(m_{0}+m_{1}\right)\right)-96\left(159-318 \nu+240 \nu^{2}-81 \nu^{3}+8 \nu^{4}\right)\right)
\end{aligned}
$$




$$
\begin{aligned}
& -21\left(210403-631209 \nu+813759 \nu^{2}-575503 \nu^{3}+219354 \nu^{4}-36804 \nu^{5}\right. \\
& \left.+1288 \nu^{6}\right)\left(m_{0}^{5}+m_{1}^{5}\right)-(1+\nu)(2 \nu-1)\left(4 2 0 \left(20281-40562 \nu+25053 \nu^{2}\right.\right. \\
& \left.-4772 \nu^{3}+52 \nu^{4}\right)\left(m_{0}^{4}+m_{1}^{4}\right)-840\left(-6407+12814 \nu-6771 \nu^{2}+364 \nu^{3}\right. \\
& \left.+436 \nu^{4}\right) m_{0} m_{1}\left(m_{0}^{2}+m_{1}^{2}\right)-2520\left(2489-4978 \nu+3837 \nu^{2}-1348 \nu^{3}\right. \\
& \left.+308 \nu^{4}\right) m_{0}^{2} m_{1}^{2}+70\left(-31607+63214 \nu+16989 \nu^{2}-48596 \nu^{3}\right. \\
& \left.+3316 \nu^{4}\right)\left(m_{0}^{3}+m_{1}^{3}\right)+12600(1+\nu)(-1+2 \nu)\left(2 \nu^{2}+35(1-\nu)\right)\left(m_{0}+m_{1}\right) \\
& \times\left(-m_{0}-m_{1}+12 m_{0} m_{1}\right)-7\left(-39599+79198 \nu-52347 \nu^{2}+12748 \nu^{3}\right. \\
& \left.\left.\left.+2452 \nu^{4}\right)\left(m_{0}+m_{1}\right)\right)+103680(1-\nu)\left(5-10 \nu+10 \nu^{2}-5 \nu^{3}+\nu^{4}\right)\right\} .
\end{aligned}
$$

One can check that the $\Psi$-function in the ground state case is reproduced if one takes $m_{0}=$ $m_{1}=0$. There is a connection of the function $\Psi$ with the integrals of motion described in [7]. In the ground state case this connection was given by the formula (10.17) of [4]:

$$
I_{2 n-1}=-i \Psi^{(p)}\left(\frac{i(2 n-1)}{2 \nu}, p\right) n(2 n-1)\left(2 \nu^{2}\right)^{n-1} p^{2 n-1} .
$$

In the case of excitations the integrals of motion are given by matrices. Let us consider the first three integrals of motion $I_{1}, I_{3}, I_{5}$. Their explicit expressions via the Virasoro generators can be found in [6].

Again let us consider an example of the excitations with $N=2$ and $k=1$ with two possibilities $I^{(+, 1)}=\{1\}, I^{(-, 1)}=\{3\}$ and $I^{(+, 1)}=\{3\}, I^{(-, 1)}=\{1\}$ which correspond to the two-dimensional descendant space spanned by two vectors $L_{-2}\left|\Delta_{+}\right\rangle$and $L_{-1}^{2}\left|\Delta_{+}\right\rangle$or their linear combinations $\left|\Delta_{+} ;\{1\},\{3\}\right\rangle,\left|\Delta_{+} ;\{3\},\{1\}\right\rangle$ given by the formula (6.4).

Let us start with the simplest case of the very first integral of motion $I_{1}$ given by the formula (11) of the paper [6]:

$$
I_{1}=L_{0}-\frac{c}{24}
$$

This operator is diagonal for the above two-dimensional space

$$
I_{1}\left(\begin{array}{l}
L_{-2}\left|\Delta_{+}\right\rangle \\
L_{-1}^{2}\left|\Delta_{+}\right\rangle
\end{array}\right)=\left(\Delta_{+}+2-\frac{c}{24}\right)\left(\begin{array}{l}
L_{-2}\left|\Delta_{+}\right\rangle \\
L_{-1}^{2}\left|\Delta_{+}\right\rangle
\end{array}\right)
$$

and

$$
\Delta_{+}+2-\frac{c}{24}=2(p \nu)^{2}+\frac{47}{24}
$$

We can easily check that we get exactly the same result if we substitute the expansion (3.26) with the coefficients given by (A.1) for $l=i /(2 \nu)$ and $m_{0}=1, m_{1}=3$ or $m_{0}=3, m_{1}=1$ into the formula (A.2) with $n=1$. We see that in this case all $\Psi_{j}^{(p)}(i /(2 \nu))=0$ with $j \geq 3$ even without fixing $m_{0}$ and $m_{1}$.

The next case of $I_{3}$ is a bit less trivial since $I_{3}$ is not diagonal any longer. Following [6], we have

$$
I_{3}=2 \sum_{n=1}^{\infty} L_{-n} L_{n}+L_{0}^{2}-\frac{c+2}{12} L_{0}+\frac{c(5 c+22)}{2880} .
$$


We see that only the first term here is not diagonal. Using the Virasoro algebra, one can check that

$$
\begin{aligned}
I_{3}\left(\begin{array}{l}
L_{-2}\left|\Delta_{+}\right\rangle \\
L_{-1}^{2}\left|\Delta_{+}\right\rangle
\end{array}\right)= & \left(\begin{array}{cc}
c & 6 \\
12 \Delta_{+} & 4
\end{array}\right)\left(\begin{array}{l}
L_{-2}\left|\Delta_{+}\right\rangle \\
L_{-1}^{2}\left|\Delta_{+}\right\rangle
\end{array}\right) \\
& +\left(8 \Delta_{+}+\left(\Delta_{+}+2\right)^{2}-\frac{(c+2)\left(\Delta_{+}+2\right)}{12}+\frac{c(5 c+22)}{2880}\right)\left(\begin{array}{l}
L_{-2}\left|\Delta_{+}\right\rangle \\
L_{-1}^{2}\left|\Delta_{+}\right\rangle
\end{array}\right) .
\end{aligned}
$$

We can diagonalize the matrix

$$
\left(\begin{array}{cc}
c & 6 \\
12 \Delta_{+} & 4
\end{array}\right)=\left(\begin{array}{cc}
C & D \\
A & B
\end{array}\right)^{-1}\left(\begin{array}{cc}
\lambda^{+} & 0 \\
0 & \lambda^{-}
\end{array}\right)\left(\begin{array}{cc}
C & D \\
A & B
\end{array}\right)
$$

with the eigenvalues

$$
\lambda^{ \pm}=-\frac{6 \nu^{2}-5(1-\nu)}{2(1-\nu)} \pm \frac{3 \sqrt{64 \nu^{2}(1-\nu)^{2} p^{2}+(1+\nu)^{2}(2 \nu-1)^{2}}}{2(1-\nu)}
$$

and the matrix elements $A, B, C, D$ taken from the definition of the vectors $\left|\Delta_{+} ;\{1\},\{3\}\right\rangle$, $\left|\Delta_{+} ;\{3\},\{1\}\right\rangle$ given by (6.4). Hence these vectors are eigenvectors of the matrix $I_{3}$. Several leading terms of $1 / p$-expansion of $A, B$ where shown in (6.7) while $C, D$ can be got from $A, B$ by changing $p \rightarrow-p$. So, for the eigenvalues of $I_{3}$ we obtain ${ }^{10}$

$$
\begin{aligned}
I_{3}^{( \pm)}= & \lambda^{ \pm}+8 \Delta_{+}+\left(\Delta_{+}+2\right)^{2}-\frac{(c+2)\left(\Delta_{+}+2\right)}{12}+\frac{c(5 c+22)}{2880} \\
\simeq & 4(p \nu)^{4}+\frac{47}{2}(p \nu)^{2} \mp 12 p \nu-\frac{4804 \nu^{2}-5769(1-\nu)}{960(1-\nu)} \\
& \mp \frac{3}{32} \frac{(1+\nu)^{2}(2 \nu-1)^{2}}{(1-\nu)^{2}}(p \nu)^{-1}+O\left(p^{-2}\right) .
\end{aligned}
$$

We can check at least for number of first orders that we get the same $1 / p$-expansion if, like in the previous case we substitute the expansion (3.26) this time for $l=3 i /(2 \nu)$ and $m_{0}=3$, $m_{1}=1$ in case of $I_{3}^{(+)}$and $m_{0}=1, m_{1}=3$ in case of $I_{3}^{(-)}$into the formula (A.2) with $n=2$. In contrast to the case of the first integral of motion $I_{1}$, the series expansion (A.4) does not terminate because of the square root in the expression (A.3) for the eigenvalues $\lambda^{ \pm}$. Also we get both even and odd powers with respect to $p$ in contrast to the case $N=0$ corresponding to the ground state.

The next case can be treated similarly. First we take $I_{5}$ from [6]

$$
\begin{aligned}
I_{5}= & \sum_{n_{1}+n_{2}+n_{3}=0}: L_{n_{1}} L_{n_{2}} L_{n_{3}}:+\sum_{n=1}^{\infty}\left(\frac{c+11}{6} n^{2}-1-\frac{c}{4}\right) L_{-n} L_{n} \\
& +\frac{3}{2} \sum_{r=1}^{\infty} L_{1-2 r} L_{2 r-1}-\frac{c+4}{8} L_{0}^{2}+\frac{(c+2)(3 c+20)}{576} L_{0}-\frac{c(3 c+14)(7 c+68)}{290304}
\end{aligned}
$$

with the normal ordering : : for which the Virasoro generators with bigger indices are placed to the right. Then using the Virasoro algebra, one can come to the following formula

$$
I_{5}\left(\begin{array}{l}
L_{-2}\left|\Delta_{+}\right\rangle \\
L_{-1}^{2}\left|\Delta_{+}\right\rangle
\end{array}\right)=5\left(\frac{5}{6}+\frac{c}{24}+\Delta_{+}\right)\left(\begin{array}{cc}
c & 6 \\
12 \Delta_{+} & 4
\end{array}\right)\left(\begin{array}{l}
L_{-2}\left|\Delta_{+}\right\rangle \\
L_{-1}^{2}\left|\Delta_{+}\right\rangle
\end{array}\right)
$$

\footnotetext{
${ }^{10}$ We hope the reader will not mix these eigenvalues $I_{j}^{( \pm)}$with the integrals of motion $I_{j}^{ \pm}$mentioned in Section 2 that equal to each other when $\kappa^{\prime}=\kappa$ and $\Delta_{+}=\Delta_{-}$.
} 


$$
\begin{aligned}
& +\left(\Delta_{+}^{3}+\frac{236-c}{8} \Delta_{+}^{2}+\left(\frac{c^{2}}{192}-\frac{227 c}{288}+\frac{3845}{72}\right) \Delta_{+}\right) \\
& +\left(\Delta_{+}^{3}-\frac{c^{3}}{13824}+\frac{1361 c^{2}}{145152}-\frac{7325 c}{5184}+\frac{221}{36}\right)\left(\begin{array}{l}
L_{-2}\left|\Delta_{+}\right\rangle \\
L_{-1}^{2}\left|\Delta_{+}\right\rangle
\end{array}\right) .
\end{aligned}
$$

We see that the matrix $I_{5}$ can be diagonalized by the same similarity transformation as $I_{3}$ because all the integrals of motion commute. The result for the asymptotic expansion of the two corresponding eigenvalues $I_{5}^{ \pm}$looks

$$
\begin{aligned}
I_{5}^{( \pm)} \simeq & 8(p \nu)^{6}+\frac{235}{2}(p \nu)^{4} \mp 120(p \nu)^{3}-\frac{4808 \nu^{2}-12503(1-\nu)}{96(1-\nu)}(p \nu)^{2} \\
& \mp \frac{15\left(4 \nu^{4}+36 \nu^{3}+21 \nu^{2}-114 \nu+57\right)}{16(1-\nu)^{2}}(p \nu) \\
& +\frac{822432 \nu^{4}+2338220 \nu^{3}-821605 \nu^{2}-3033230 \nu+1516615}{96768(1-\nu)^{2}}+O\left(p^{-1}\right) .
\end{aligned}
$$

Again this result perfectly matches the expansion of (A.2) for $n=3$ with $\Psi^{(p)}(5 i /(2 \nu), p)$ taken for $m_{0}=3, m_{1}=1$ in case of $I_{5}^{(+)}$and for $m_{0}=1, m_{1}=3$ in case of $I_{5}^{(-)}$. Similar to $I_{3}^{( \pm)}$, the integral $I_{5}^{( \pm)}$and all further integrals $I_{2 n-1}^{( \pm)}$are not polynomials with respect to $p$ in contrast to the ground state case.

\section{B The functions $\overline{\boldsymbol{F}}(\boldsymbol{x}, \boldsymbol{p})$ and $\boldsymbol{x}_{r}^{ \pm}(\boldsymbol{p})$}

In Section 3 we discussed several functions defined within the TBA approach. In the previous appendix we showed few orders of the function $\Psi^{(p)}(l, p)$. We also need the functions $\bar{F}(x, p)$ and $x_{r}^{ \pm}(p)$. As was described in Section 3, the asymptotic expansions of these functions and the function $\Psi^{(p)}(l, p)$ are calculated order by order with respect to $1 / p$ via the iterative procedure. There we explained how does the very first iteration work. Once the function $\Psi^{(p)}(l, p)$ is found up to some order, the next order of the function $\bar{F}(x, p)$ can be obtained via the equation (3.17). The coefficients of (3.18) are determined from the equation (3.21). The further iteration steps are straightforward but the answer becomes rather cumbersome already after several iterations. For the reader who wants to check his own calculations we show few orders of the expansions (3.23) and (3.18) in the case $k=1$. We do not think it would be instructive to show further orders. As in the previous appendix we use the shorthand notation $m_{0} \equiv I_{1}^{(+, 1)}, m_{1} \equiv I_{1}^{(-, 1)}$.

$$
\begin{aligned}
\bar{F}_{0}(x)= & -i \frac{(1+\nu)(2 \nu-1)}{576 \nu(1-\nu)}\left(1-12\left(m_{0}+m_{1}\right)\right)-i x^{2} \frac{2 \nu^{2}+11(1-\nu)}{48 \nu(1-\nu)} \\
\bar{F}_{1}(x)= & i \frac{(1+\nu)(2 \nu-1)\left(2 \nu^{2}+23(1-\nu)\right)}{9216 \nu^{2}(1-\nu)^{2}}-x \frac{(1+\nu)^{2}(2 \nu-1)^{2}}{27648 \nu^{2}(1-\nu)^{2}}\left(1-12\left(m_{0}+m_{1}\right)\right) \\
& +x^{3} \frac{4 \nu^{4}-44 \nu^{3}+309 \nu^{2}-530 \nu+265}{6912 \nu^{2}(1-\nu)^{2}}, \\
\bar{F}_{2}(x)= & -\frac{i(1+\nu)(2 \nu-1)}{2388787200 \nu^{3}(1-\nu)^{3}}\left(120\left(436 \nu^{4}+364 \nu^{3}-6771 \nu^{2}+12814 \nu-6407\right)\right. \\
& \times\left(m_{0}^{3}+m_{1}^{3}\right)+3600(1+\nu)(2 \nu-1)\left(2 \nu^{2}+23(1-\nu)\right)\left(m_{0}+m_{1}\right) \\
& \left.\times\left(1-6\left(m_{0}+m_{1}\right)\right)+2452 \nu^{4}+9148 \nu^{3}-50547 \nu^{2}+82798 \nu-41399\right) \\
& +\frac{x(1+\nu)(2 \nu-1)}{3317760 \nu^{3}(1-\nu)^{3}}\left(556 \nu^{4}-1676 \nu^{3}+2859 \nu^{2}-2366 \nu+1183\right)\left(m_{0}^{2}-m_{1}^{2}\right)
\end{aligned}
$$




$$
\begin{aligned}
& -\frac{i x^{2}(1+\nu)(2 \nu-1)}{19906560 \nu^{3}(1-\nu)^{3}}\left(556 \nu^{4}-2036 \nu^{3}+3039 \nu^{2}-2006 \nu+1003\right) \\
& \times\left(1-12\left(m_{0}+m_{1}\right)\right)-\frac{i x^{4}}{9953280 \nu^{3}(1-\nu)^{3}}\left(1112 \nu^{6}-2796 \nu^{5}-5154 \nu^{4}\right. \\
& \left.+64603 \nu^{3}-154059 \nu^{2}+146109 \nu-48703\right), \\
& \bar{F}_{3}(x)=-i \frac{(1+\nu)(2 \nu-1)}{15288238080 \nu^{4}(1-\nu)^{4}}\left(m_{0}^{2}-m_{1}^{2}\right)\left(3 \left(7736 \nu^{6}-49644 \nu^{5}+41430 \nu^{4}\right.\right. \\
& \left.+233059 \nu^{3}-658107 \nu^{2}+649893 \nu-216631\right)\left(m_{0}^{2}+m_{1}^{2}\right) \\
& -4(1+\nu)(2 \nu-1)\left(932 \nu^{4}+788 \nu^{3}-22227 \nu^{2}+42878 \nu-21439\right) \\
& \left.\times\left(1-12\left(m_{0}+m_{1}\right)\right)\right)+x \frac{(1+\nu)(2 \nu-1)}{229323571200 \nu^{4}(1-\nu)^{4}}\left(1 0 8 0 \left(1496 \nu^{6}-12924 \nu^{5}\right.\right. \\
& \left.+42270 \nu^{4}-75161 \nu^{3}+78753 \nu^{2}-49407 \nu+16469\right)\left(m_{0}^{3}+m_{1}^{3}\right) \\
& -960(1+\nu)(2 \nu-1)\left(556 \nu^{4}-1676 \nu^{3}+2859 \nu^{2}-2366 \nu+1183\right)\left(m_{0}+m_{1}\right) \\
& \times\left(1-6\left(m_{0}+m_{1}\right)\right)+138728 \nu^{6}-926052 \nu^{5}+2802450 \nu^{4}-4743023 \nu^{3} \\
& \left.+4847079 \nu^{2}-2970681 \nu+990227\right)+i x^{2} \frac{(1+\nu)(2 \nu-1)}{637009920 \nu^{4}(1-\nu)^{4}}\left(m_{0}^{2}-m_{1}^{2}\right) \\
& \times\left(4568 \nu^{6}-40572 \nu^{5}+138270 \nu^{4}-239513 \nu^{3}+230049 \nu^{2}-132351 \nu+44117\right) \\
& +x^{3} \frac{(1+\nu)^{2}(2 \nu-1)^{2}}{5733089280 \nu^{4}(1-\nu)^{4}}\left(2284 \nu^{4}-8084 \nu^{3}+12111 \nu^{2}-8054 \nu+4027\right) \\
& \times\left(1-12\left(m_{0}+m_{1}\right)\right)+\frac{x^{5}}{4777574400 \nu^{4}(1-\nu)^{4}}\left(9136 \nu^{8}-76576 \nu^{7}+196840 \nu^{6}\right. \\
& \left.+149096 \nu^{5}-3382325 \nu^{4}+10503740 \nu^{3}-14391926 \nu^{2}+9334868 \nu-2333717\right), \\
& \bar{F}_{4}(x)=\frac{i(1+\nu)(2 \nu-1)}{2157476157849600 \nu^{5}(1-\nu)^{5}}\left(6 0 4 8 \left(4048 \nu^{8}+208064 \nu^{7}-1145688 \nu^{6}+1757040 \nu^{5}\right.\right. \\
& \left.+1432457 \nu^{4}-8416452 \nu^{3}+12210950 \nu^{2}-8061828 \nu+2015457\right)\left(m_{0}^{5}+m_{1}^{5}\right) \\
& +3809872 \nu^{8}-215990816 \nu^{7}+939983096 \nu^{6}-1142084632 \nu^{5}-1598906179 \nu^{4} \\
& +6538494484 \nu^{3}-8737091978 \nu^{2}+5620794700 \nu-1405198675 \\
& -196(1+\nu)(2 \nu-1)\left(m_{0}+m_{1}\right)\left(2 4 0 \left(11528 \nu^{6}-109308 \nu^{5}+124842 \nu^{4}+705307 \nu^{3}\right.\right. \\
& \left.-2193591 \nu^{2}+2209125 \nu-736375\right)\left(m_{0}^{3}+m_{1}^{3}\right)+120\left(27112 \nu^{6}-287566 \nu^{5}\right. \\
& \left.+627666 \nu^{4}+187193 \nu^{3}-2262129 \nu^{2}+2602239 \nu-867413\right) m_{0} m_{1}\left(m_{0}^{2}+m_{1}^{2}\right) \\
& +10\left(247064 \nu^{6}+795588 \nu^{5}-9542418 \nu^{4}+12330151 \nu^{3}+6743697 \nu^{2}-15490527 \nu\right. \\
& +5163509)\left(m_{0}^{2}+m_{1}^{2}\right)+10\left(575464 \nu^{6}+728508 \nu^{5}-17201838 \nu^{4}+25221881 \nu^{3}\right. \\
& \left.+6701007 \nu^{2}-23174337 \nu+7724779\right) m_{0} m_{1}+360\left(2 \nu^{2}+23(1-\nu)\right)\left(676 \nu^{4}\right. \\
& \left.-3716 \nu^{3}+12489 \nu^{2}-17546 \nu+8773\right) m_{0} m_{1}\left(m_{0}+m_{1}\right)-5040(1+\nu)(2 \nu-1) \\
& \times\left(68 \nu^{4}+92 \nu^{3}-2223 \nu^{2}+4262 \nu-2131\right)\left(m_{0}+m_{1}\right)+7\left(35272 \nu^{6}\right. \\
& \left.\left.\left.-272436 \nu^{5}+362346 \nu^{4}+559733 \nu^{3}-2128749 \nu^{2}+2218659 \nu-739553\right)\right)\right) \\
& -\frac{x(1+\nu)(2 \nu-1)}{1284211998720 \nu^{5}(1-\nu)^{5}}\left(m_{0}^{2}-m_{1}^{2}\right)\left(8 \left(114704 \nu^{8}-186848 \nu^{7}-1703896 \nu^{6}\right.\right. \\
& \left.+8391976 \nu^{5}-17873683 \nu^{4}+22437364 \nu^{3}-17519338 \nu^{2}+8605900 \nu-2151475\right) \\
& \times\left(m_{0}^{2}+m_{1}^{2}\right)+7(1+\nu)(2 \nu-1)\left(11240 \nu^{6}-137412 \nu^{5}+480594 \nu^{4}-893735 \nu^{3}\right.
\end{aligned}
$$




$$
\begin{aligned}
& \left.\left.+965295 \nu^{2}-622113 \nu+207371\right)\left(1-12\left(m_{0}+m_{1}\right)\right)\right) \\
& -i \frac{x^{2}(1+\nu)^{2}(2 \nu-1)^{2}}{77052719923200 \nu^{5}(1-\nu)^{5}}\left(1 2 0 \left(1247080 \nu^{6}-6770580 \nu^{5}+15390258 \nu^{4}\right.\right. \\
& \left.-18566683 \nu^{3}+12601659 \nu^{2}-3981981 \nu+1327327\right)\left(m_{0}^{3}+m_{1}^{3}\right) \\
& +1680\left(4568 \nu^{6}-40572 \nu^{5}+138270 \nu^{4}-239513 \nu^{3}+230049 \nu^{2}-132351 \nu\right. \\
& +44117)\left(m_{0}+m_{1}\right)\left(1-6\left(m_{0}+m_{1}\right)\right)+7\left(1201400 \nu^{6}-6364860 \nu^{5}+14007558 \nu^{4}\right. \\
& \left.\left.-16171553 \nu^{3}+10301169 \nu^{2}-2658471 \nu+886157\right)\right) \\
& +\frac{x^{3}(1+\nu)(2 \nu-1)}{321052999680 \nu^{5}(1-\nu)^{5}}\left(m_{0}^{2}-m_{1}^{2}\right)\left(2622064 \nu^{8}-14133568 \nu^{7}+33585976 \nu^{6}\right. \\
& \left.-49220560 \nu^{5}+55866331 \nu^{4}-52813996 \nu^{3}+43115890 \nu^{2}-21866764 \nu+5466691\right) \\
& -\frac{i x^{4}(1+\nu)(2 \nu-1)}{3852635996160 \nu^{5}(1-\nu)^{5}}\left(2622064 \nu^{8}-14517280 \nu^{7}+35135944 \nu^{6}\right. \\
& \left.-51550552 \nu^{5}+56686759 \nu^{4}-51453364 \nu^{3}+41083762 \nu^{2}-20513692 \nu+5128423\right) \\
& \times\left(1-12\left(m_{0}+m_{1}\right)\right)-\frac{4815794995200 \nu^{5}(1-\nu)^{5}}{45244128 \nu^{1} 0-25645072 \nu^{9}} \\
& +45932736 \nu^{8}-30480936 \nu^{7}+22676526 \nu^{6}-291308781 \nu^{5}+1140456861 \nu^{4} \\
& \left.-2039726202 \nu^{3}+1941710088 \nu^{2}-954519025 \nu+190903805\right) .
\end{aligned}
$$

For the parameters $x_{1}^{ \pm}(p) \equiv x^{ \pm}(p)=\sum_{j=0}^{\infty} x_{j}^{ \pm} p^{-j}$ which determine the Bethe roots via the relation (3.18) we had the initial conditions $x_{0}^{+}=\frac{i m_{0}}{2}, x_{0}^{-}=-\frac{i m_{1}}{2}$ and then

$$
\begin{aligned}
x_{1}^{+}= & \frac{i\left(2 \nu^{2}+11(1-\nu)\right)}{192 \nu(1-\nu)} m_{0}^{2}-\frac{i(1+\nu)(2 \nu-1)}{576 \nu(1-\nu)}\left(1-12\left(m_{0}+m_{1}\right)\right), \\
x_{2}^{+}= & \frac{i\left(20 \nu^{4}-220 \nu^{3}+681 \nu^{2}-922 \nu+461\right) m_{0}^{3}}{55296 \nu^{2}(1-\nu)^{2}} \\
& +\frac{i(1+\nu)(2 \nu-1)\left(2 \nu^{2}+23(1-\nu)\right)}{55296 \nu^{2}(1-\nu)^{2}}\left(-m_{0}+18 m_{0}^{2}+12 m_{0} m_{1}-6 m_{1}^{2}\right), \\
x_{3}^{+}= & \frac{i\left(1288 \nu^{6}-36804 \nu^{5}+219354 \nu^{4}-575503 \nu^{3}+813759 \nu^{2}-631209 \nu+210403\right) m_{0}^{4}}{159252480 \nu^{3}(1-\nu)^{3}} \\
& +\frac{i(1+\nu)(2 \nu-1)\left(52 \nu^{4}-4772 \nu^{3}+25053 \nu^{2}-40562 \nu+20281\right) m_{0}^{3}}{9953280 \nu^{3}(1-\nu)^{3}} \\
& -\frac{i(1+\nu)(2 \nu-1)\left(436 \nu^{4}+364 \nu^{3}-6771 \nu^{2}+12814 \nu-6407\right) m_{1}\left(3 m_{0}^{2}+m_{1}^{2}\right)}{19906560 \nu^{3}(1-\nu)^{3}} \\
& +\frac{i(1+\nu)^{2}(2 \nu-1)^{2}\left(2 \nu^{2}+35(1-\nu)\right)\left(-m_{0}-m_{1}+12 m_{0} m_{1}+6 m_{1}^{2}\right)}{663552 \nu^{3}(1-\nu)^{3}} \\
& +\frac{i(1+\nu)(2 \nu-1)\left(3316 \nu^{4}-48596 \nu^{3}+16989 \nu^{2}+63214 \nu-31607\right) m_{0}^{2}}{79626240 \nu^{3}(1-\nu)^{3}} \\
& -\frac{i(1+\nu)(2 \nu-1)\left(308 \nu^{4}-1348 \nu^{3}+3837 \nu^{2}-4978 \nu+2489\right) m_{0} m_{1}^{2}}{3317760 \nu^{3}(1-\nu)^{3}} \\
& -\frac{i(1+\nu)(2 \nu-1)\left(2452 \nu^{4}+12748 \nu^{3}-52347 \nu^{2}+79198 \nu-39599\right)}{2388787200 \nu^{3}(1-\nu)^{3}},
\end{aligned}
$$




$$
\begin{aligned}
x_{4}^{+}= & \frac{i}{458647142400 \nu^{4}(1-\nu)^{4}}\left(-3\left(18704 \nu^{8}+1330336 \nu^{7}-16442440 \nu^{6}+75087544 \nu^{5}\right.\right. \\
& \left.-183046975 \nu^{4}+267969460 \nu^{3}-244932514 \nu^{2}+133379452 \nu-33344863\right) m_{0}^{5} \\
& -150(1+\nu)(2 \nu-1)\left(3256 \nu^{6}+51156 \nu^{5}-738570 \nu^{4}+2884979 \nu^{3}-5217867 \nu^{2}\right. \\
& +4530453 \nu-1510151) m_{0}^{4}+30(1+\nu)(2 \nu-1)\left(53816 \nu^{6}-1086444 \nu^{5}+6642390 \nu^{4}\right. \\
& \left.-7848221 \nu^{3}-4235067 \nu^{2}+9791013 \nu-3263671\right) m_{0}^{3}-90(1+\nu)(2 \nu-1)\left(7736 \nu^{6}\right. \\
& \left.-49644 \nu^{5}+41430 \nu^{4}+233059 \nu^{3}-658107 \nu^{2}+649893 \nu-216631\right) m_{1}\left(4 m_{0}^{3}-m_{1}^{3}\right) \\
& +7200(1+\nu)^{2}(2 \nu-1)^{2}\left(2 \nu^{2}+23(1-\nu)\right)^{2}\left(-3 m_{0}^{2}-2 m_{0} m_{1}+36 m_{0}^{2} m_{1}+m_{1}^{2}\right. \\
& \left.-12 m_{1}^{3}\right)+(1+\nu)(2 \nu-1)\left(74632 \nu^{6}-808308 \nu^{5}+3707610 \nu^{4}-8492107 \nu^{3}\right. \\
& \left.+10979811 \nu^{2}-8080509 \nu+2693503\right) m_{0}-60(1+\nu)(2 \nu-1)\left(9976 \nu^{6}-100044 \nu^{5}\right. \\
& \left.\left.+431430 \nu^{4}-1092901 \nu^{3}+1621773 \nu^{2}-1290387 \nu+430129\right) m_{0} m_{1}^{2}\left(3 m_{0}-2 m_{1}\right)\right) .
\end{aligned}
$$

The coefficients $x_{j}^{-}$can be got from $\tilde{x}_{j}^{+}$obtained from $x_{j}^{+}$by the replacement $m_{0} \leftrightarrow m_{1}$ as follows:

$$
x_{2 j}^{-}=-\tilde{x}_{2 j}^{+}, \quad x_{2 j+1}^{-}=\tilde{x}_{2 j+1}^{+}, \quad j=0,1, \ldots
$$

\section{The function $\omega^{\text {sc }}$}

In Section 4 we explained how we determine our main object: the function $\omega^{\mathrm{sc}}$. The asymptotic expansion for $\omega^{\mathrm{sc}}$ is related to the function $\Theta$ by the formula (4.9). In it's turn the coefficients $\Theta_{n}$ of asymptotic expansion (4.6) of the function $\Theta$

$$
\Theta(l, m \mid p, \alpha) \simeq \sum_{j=0}^{\infty} \Theta_{j}(l, m \mid \alpha) p^{-j}
$$

can be found with help of the TBA data of Section 3 and Appendices A, B via solving the equation (4.7) by iterations. In this appendix we show the result for several leading coefficients again in the case $k=1$. With the exception of the coefficient $\Theta_{0}$, all other coefficients $\Theta_{j}$ are polynomials with respect to $l$ and $m$ :

$$
\begin{aligned}
\Theta_{0}(l, m \mid \alpha)= & -\frac{i}{l+m}, \\
\Theta_{1}(l, m \mid \alpha)= & 0 \\
\Theta_{2}(l, m \mid \alpha)= & -\frac{\nu^{2} \alpha(2-\alpha)+2 i(1-\nu)(-i+2 \nu(l+m))}{96 \nu(1-\nu)}\left(1-12\left(m_{0}+m_{1}\right)\right), \\
\Theta_{3}(l, m \mid \alpha)= & -\frac{m_{0}^{2}-m_{1}^{2}}{512 \nu^{2}(1-\nu)^{2}}\left(-3 \nu^{4} \alpha^{2}(2-\alpha)^{2}-24 i \alpha(2-\alpha)(1-\nu) \nu^{2}(-i+\nu(l+m))\right. \\
& \left.+32(1-\nu)^{2}(-i+\nu(l+m))(-i+2 \nu(l+m))\right) \\
\Theta_{4}(l, m \mid \alpha)= & -\frac{1}{184320 \nu^{3}(1-\nu)^{3}}\left(-5 \nu^{6}\left(5\left(m_{0}^{3}+m_{1}^{3}\right)-42\left(m_{0}+m_{1}\right)^{2}+7\left(m_{0}+m_{1}\right)\right)\right. \\
& \times \alpha^{3}(2-\alpha)^{3}+10 i \nu^{4}(1-\nu)\left(-24(-3 i+2(l+m) \nu)\left(m_{0}^{3}+m_{1}^{3}\right)\right. \\
& \left.+2 m^{(0,1)}(-16 i+9(l+m) \nu)-(-i+(l+m) \nu)\right) \alpha^{2}(2-\alpha)^{2}+8 \nu(1-\nu) \\
& \times\left(2 \nu(1-\nu)\left(7+120\left(m_{0}^{3}+m_{1}^{3}\right)\right)(-i+(l+m) \nu)^{2}-3 \nu(1-\nu)\left(1+10 m^{(0,1)}\right)\right. \\
& \times(-i+2 l \nu)(-i+2 m \nu)-20 i \nu(1+\nu)(2 \nu-1)(-i+(l+m) \nu) m^{(0,1)}
\end{aligned}
$$




$$
\begin{aligned}
& \left.-i \nu(-i+(l+m) \nu)\left(4 \nu^{2}+5\left(1+24\left(m_{0}^{3}+m_{1}^{3}\right)\right)(1-\nu)\right)\right) \alpha(2-\alpha) \\
& -16 i \nu^{4}(1-\nu)(2-\nu)\left(1+10 m^{(0,1)}\right)(l-m) \alpha(1-\alpha)(2-\alpha)+8 i(1-\nu)^{3} \\
& \times\left(7+120\left(m_{0}^{3}+m_{1}^{3}\right)\right)(-i+(l+m) \nu)(-i+2(l+m) \nu)(-3 i+2(l+m) \nu) \\
& +8(1-\nu)^{2}(1+\nu)(2 \nu-1)\left(1+10 m^{(0,1)}\right)(2(-i+(l+m) \nu)(-i+2(l+m) \nu) \\
& +(-i+2 l \nu)(-i+2 m \nu)))
\end{aligned}
$$

where $m^{(0,1)}=m_{0}^{3}+m_{1}^{3}+6\left(m_{0}+m_{1}\right)^{2}-m_{0}-m_{1}$. Unfortunately, we cannot show all the orders in the expansion of $\Theta$ and other functions from the previous Appendices that we needed in order to get the formulae (6.9)-(6.11) because the expressions become very cumbersome with growing order. Again the ground state result can be obtained by taking $m_{0}=m_{1}=0$.

\section{Acknowledgements}

Our special thanks go to M. Jimbo, T. Miwa and F. Smirnov with whom the work on this paper was started. Also we would like to thank F. Göhmann, A. Klümper and S. Lukyanov for many stimulating discussions. We are grateful to the Volkswagen Foundation for financial support.

\section{References}

[1] Boos H., Jimbo M., Miwa T., Smirnov F., Takeyama Y., Hidden Grassmann structure in the XXZ model, Comm. Math. Phys. 272 (2007), 263-281, hep-th/0606280.

[2] Boos H., Jimbo M., Miwa T., Smirnov F., Takeyama Y., Hidden Grassmann structure in the XXZ model. II. Creation operators, Comm. Math. Phys. 286 (2009), 875-932, arXiv:0801.1176.

[3] Jimbo M., Miwa T., Smirnov F., Hidden Grassmann structure in the XXZ model. III. Introducing Matsubara direction, J. Phys. A: Math. Theor. 42 (2009), 304018, 31 pages.

[4] Boos H., Jimbo M., Miwa T., Smirnov F., Hidden Grassmann structure in the XXZ model. IV. CFT limit, Comm. Math. Phys. 299 (2010), 825-866, arXiv:0911.3731.

[5] Destri C., de Vega H.J., Unified approach of thermodynamic Bethe ansatz and finite size corrections for lattice models and field theories, Nuclear Phys. B 438 (1995), 413-454, hep-th/9407117.

[6] Bazhanov V., Lukyanov S., Zamolodchikov A., Integrable structure of conformal field theory, quantum KdV theory and thermodynamic Bethe ansatz, Comm. Math. Phys. 177 (1996), 381-398, hep-th/9412229.

[7] Bazhanov V., Lukyanov S., Zamolodchikov A., Integrable structure of conformal field theory II. $Q$-operator and DDV equation, Comm. Math. Phys. 190 (1997), 247-278, hep-th/9604044.

[8] Bazhanov V., Lukyanov S., Zamolodchikov A., Integrable structure of conformal field theory. III. The Yang-Baxter relation, Comm. Math. Phys. 200 (1999), 297-324, hep-th/9805008.

[9] Baxter R.J., Exactly solved models in statistical mechanics, Academic Press, Inc., London, 1982.

[10] Zamolodchikov A.B., Higher order integrals of motion in two-dimensional models of the field theory with a broken conformal symmetry, JETP Lett. 46 (1987), 160-164.

[11] Boos H., Jimbo M., Miwa T., Smirnov F., Completeness of a fermionic basis in the homogeneous XXZ model, J. Math. Phys. 50 (2009), 095206, 10 pages, arXiv:0903.0115.

[12] Suzuki M., Transfer-matrix method and Monte Carlo simulation in quantum spin systems, Phys. Rev. B 31 (1985), 2957-2965.

[13] Boos H., Göhmann F., On the physical part of the factorized correlation functions of the XXZ chain, J. Phys. A: Math. Theor. 42 (2009), 315001, 27 pages, arXiv:0903.5043.

[14] Jimbo M., Miwa T., Smirnov F., On one-point functions of descendants in sine-Gordon model, arXiv:0912.0934.

[15] Jimbo M., Miwa T., Smirnov F., Hidden Grassmann structure in the XXZ model. V. Sine-Gordon model, arXiv:1007.0556.

[16] Dotsenko V.S., Fateev V.A., Conformal algebra and multipoint correlator functions in 2D statistical models, Nuclear Phys. B 240 (1984), 312-348. 
[17] Gaudin M., Thermodynamics of the Heisenberg-Ising ring for $\Delta>\sim 1$, Phys. Rev. Lett. 26 (1971), 13011304.

Takahashi M., One-dimensional Heisenberg model at finite temperature, Prog. Theor. Phys. 46 (1971), 401-415.

Takahashi M., Suzuki M., One-dimensional anisotropic Heisenberg model at finite temperatures, Prog. Theor. Phys. 46 (1972), 2187-2209.

Woynarovich F., Excitation spectrum of the spin-1/2 Heisenberg chain and conformal invariance, Phys. Rev. Lett. 59 (1987), 259-261.

de Vega H.J., Woynarovich F., Method for calculating finite size corrections in Bethe ansatz systems: Heisenberg chain and six-vertex model, Nuclear Phys. B 251 (1985), 439-456.

[18] Bazhanov V., Lukyanov S., Zamolodchikov A., Higher-level eigenvalues of Q-operators and Schrödinger equation, Adv. Theor. Math. Phys. 7 (2003), 711-725, hep-th/0307108.

[19] Klümper A., Batchelor M., Pearce P.A., Central charges of the 6- and 19-vertex models with twisted boundary conditions, J. Phys. A: Math. Gen. 24 (1991), 3111-3133.

[20] Klümper A., Free energy and correlation length of quantum chains related to restricted solid-on-solid lattice models, Ann. Physik (8) 1 (1992), 540-553. 